\title{
XXI.
}

\section{Ueber die Heilung aseptischer Schnittwunden der menschlichen Haut.}

\author{
Von Dr. Otto Busse, \\ II. Assistenten am Pathologischen Iustitut der Universität Greifswald.
}

(Hierzu Taf. VIII.)

In der Heilung von Wunden giebt es vor Allem drei Punkte, die noch der Aafklärung bedürfen, und über die trotz einer Reihe von Arbeiten aus der neuesten Zeit keine Einigung erzielt worden ist. Es sind dies die Fragen:

1) Woraus besteht und bildet sich die die Wundränder verklebende Masse?

2) Welchen Antheil haben die weissen Blutkörperchen an den Heilungsvorgängen?

3) Welche Rolle spielt die Grundsubstanz bei den Gewebsveränderungen?

Bezüglich des ersten Punktes handelt es sich darum, ob das die Wundränder schon wenige Stunden wach der Verletzung vereinigende Netzwerk ein Fibrin-Exsudat ist, oder ob sich das Gewebe selbst und in Substanz etwa an der Bildung des fibrinähnlichen Maschenwerkes betheiligt. Letztere Ansicht hatte besonders Thiersch ${ }^{1}$ ) gestützt auf eine Reihe von wichtigen Beobachtungen und gründlichen eigenen Untersuchungen vertreten. Ihm stehen die meisten der späteren Forscher gegen. über. Thiersch entscheidet sich erst, nachdem alle gebräuchlichen Theorien durchgeprüft und zur Auslegung der Bilder vergeblich herangezogen sind, für seine neue Ansicht, indem er seinen Beweis folgendermaassen begründet:

"Trennt man die frischverklebten Wundflächen, so geht

1) Thierseb, Ueber die feineren anatomischen Veränderungen nach Verwundung der Weichtheile. Handbuch der allgemeinen und speciellen Chirurgie von Pitha und Billroth. 1867. 

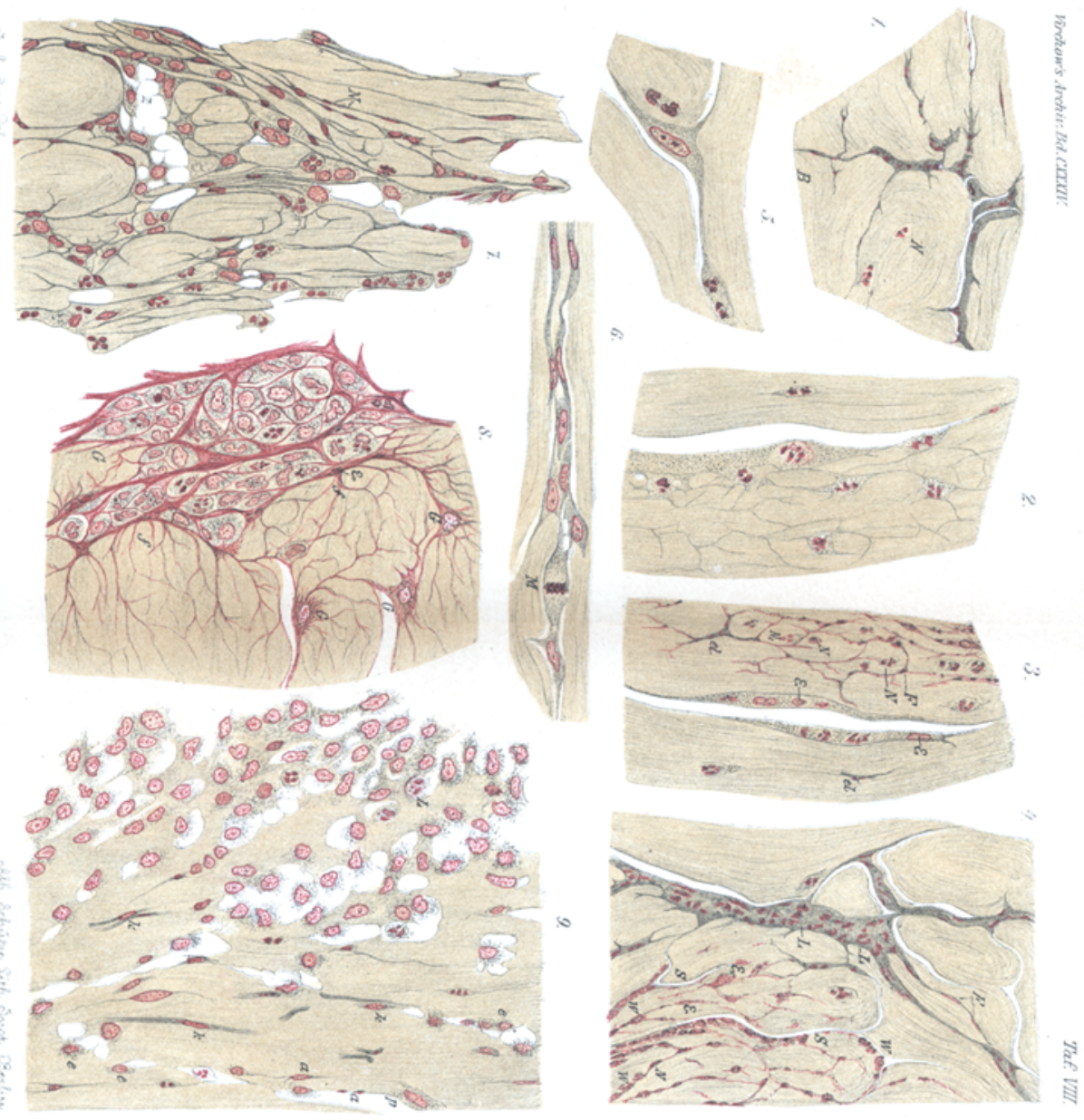

$\frac{\text { s }}{\text { S }}$ 
die Trenangslinie mitten Aurch die vermentiche Zwishensubstanz. Wäre sie ein Blutgerinnsel, so würde dasselbe gewiss das eine oder andere Mal dor einen Wundläche, ohne aich in der Mitte gespalten za haben, ganz anhaften. Wollte man annehroen, dass die Wundlächen von vornherein sich mit einer Schicht geronnenen Blutes bedeckten, und dass diese beiden Sohichten später in der Mittellinie verklebten, so dass hier der Zusammenhang ein geringerer wäre, so spricht dagegen, arstens, dass die Wundfluchen niemals in dieser Weise getrennt waren, um sich jede für sich mit geronnenem Blute zu überziehen und zweitens, dass der Versuch die der Wundläche anhaftende Gerinnung, die am frischen Präparat einen zarten röthlichen gallertigen Saum bildet, unter dem Mikroskop mit Nadeln abzulösen, immer misslingt; sowie ein Stück abreisst, so folgt dem Rissstick ein Theil Gewebe, welches durch Gehalt an Muskel oder Fett oder sonstwie charakterisirt ist. Dies Verhalten beim Abreissen spricht aber zugleich gegen die ausserdem noch mögliche Annahme, dass jede Wundfläche sich mit einer Schicht gerinnenden Exsudates bedeckt habe, die dann an ihren Berührungsflächen verklebt seien.

Wenn aber weder geronnenes Blut noch Exsudatfaserstoff, so kann die vermeintliche Zwischensubstanz nichts anderes sein, als das mit Blut getränkte ontzündlich infiltrirte Bindegewebe der Wundflächen selbst. In diesem Falle ist das Blut in der Bindegewebssubstanz selbst eingelagert und die feinkörnige Gem 3.imung, von der jedes Blutkörperchen eingekapselt ist, beruht suf einer Gerinnung in dem mit Blutplasma gemengten parenchymatösen Safte. Die bogenförmig verlaufenden Linien sind dann Begrenzungslinien einzelner Bindegewebs-Abschnitte."

Schon im nächsten Jahre erschien von E. Aufrecht ${ }^{1}$ ) ein Aufsatz, der im Gegensatz zu Thiersch die Verklebungsschicht für homogenes, dem Blutfibrin chemisch und physikalisch völlig gleichstehendes Fibrin erklärt; die stärker hervortretenden sich vielfach kreuzenden Linien sind Falten der glasigen Fibrinmasse.

3) Aufrecht, Ueber die Genese des Bindegewebes nebs einigen Bemerkungen über die Neubildung quergestreifter Muskelfasem und die Heilung per primam intentionem. Dieses Archiv. Bd. 44, 1868. 
Auch Gussenbauer ${ }^{1}$ ) führte in ähnlicher Weise aus, dass die Verklebung bewirkt wird 1) bei Hornhautwunden durch den parenchymatösen Saft des Hornhautgewebes, 2) bei Knorpelwunden durch die Gewebsflüssigkeit und das aus den eventuell durchschnittenen Gefässen austretende Blnt und 3) bei Wunden im blutführenden Gewebe durch Blut und interstitielle Gewebsflüssigkeit. Einen Hauptbeweis gegen Thiersch erblickt Gussenbauer in dem Umstande, dass in der Verklebungsmasse keine Bindegewebskörperchen vorhanden seien, die man nothwendigerweise antreffen müsse, wenn anders jene wirklich durch Veränderung des Bindegewebes entstanden sei.

Der Ansicht dieser beiden Autoren folgend erklären neuere Forscher die fragliche Substanz für Fibrinexsudat, ohne eine Begründung für eine derartige Entscheidung anzugeben. Eine Reibe hierher gehörender Arbeiten sind in dem letzten Jahrzehnt unter Ziegler ${ }^{2}$ ) ausgeführt worden.

Dieselben nehmen auch in Bezug auf den zweiten oben erwähnten Punkt, der von der Antheilnahme der Leukocyten an den Heilungsvorgängen handelt, eine entgegengesetzte Stellung ein wie Thiersch. Dieser sprach sich folgendermaassen aus: „Die Zellen in der Bildung (der Verklebungsmasse) werden zunächst wie in den Randtheilen des Präparates ebenfalls, dem Bindegewebe angehören, ohne dass damit ausgeschlossen ist, dass auch die dem ergossenen Blute angehörigen farblosen Blutkörperchen, an denen es gewiss nicht mangelt, an der Proliferation dieser Zellen sich betheiligen. Da aber solcher farbloser Blutkörperchen im Vergleich mit den Bindesubstanzzellen immer nur wenige sein werden, und da man die neuen Zellen in grosser

1) C. Gussenbauer, Ueber die Heilung per primam intentionem. Arch. für klin. Chir., herausgegeb. v. Langenbeck. Bd. XII. 1871. S. 791.

3) Kraft, Zur Histogenese des periostalen Callus. Ziegler's Beiträge. Bd. 1. Heft 1. 1886. - Podwyssozky, Experimentelle Untersuchungen über die Regeneration der Drüsengewebe. Ziegler's Beitr. Bd. 1. 1886 u. Bd. 2. 1887. - Coën, Beiträge zur normalen und pathologischen Histologie der Milchdräse. Ziegler's Beitr. Bd. 2. - otto Fischer, Experimentelle Untersuchungen über die Heilung von Schnittwunden der Haut. Inaug.-Diss. Tübingen 1888. - Nik if or off, Untersuchungen über den Bau und die Entwickelungsgeschichte des Granulationsgewebes. Ziegler's Beitr. 1890. Bd. 8. S. 400. 
Menge auch an Orten antrift, wo das Biut nicht hingelangt ist, so glaube ich nicht, dass ein beträchtlicher Theil der neven Zellen von den farblosen Bluttoôrperchen abstammat."

Auch die Eiterkörperchen hält Thiersch für Abkömmlinge der Gewebszellen. Er charakterisirt das Verhältniss der beiden mit Solgenden Worten:

"Dabei mache ich den Unterschied zwischen Granulationszelle und Eiterkörperchen, dass jederzeit eine Granulationszelle sich in eine Eiterzelle umzuwandeln vermag, aber nicht umgekehrt.

Das Resultat, zu dem ihn der Vergleich der Hellung mit und ohne Eiterung führt ist das,

"dass in histogenetischer Beziohung zwischen MejLng ohne Eiterung und Heilung mit Eiterung kein anderer Unterschied besteht, als dass bei der Eiterung ein Theil der zellig amgewandelten Gewobe statt organisatorisch fixirt zu werden, durch Verflussigung der intercellulären Substanz zur Ablösung von den sich behauptenden Geweben gelangt."

A ufrecht hielt jede Rundzelle in den Präparaten für einen Leukocyten und lässt durch diese das nene Gewebe gebildet werden.

In den neuesten Arbeiten besonders denen von Fischer und Nikiforoff werden nur die in den ersten Tagen zahlreich vorhandenen Rundzellei für ausgewanderte farblose Blutkörperchen gehalten, diesen gesellon sich später Ablömmlinge der Gewebszellen zu, die dann als Fibroblasten die eigentliche Heilung, $_{3}$.h. die Bildung der Narbe übernehmen. Wir vermissen in diesen Arbeiten, dass diese Ansichten, die in einen bewussten Gegensatz zu den wohlbegründeten Resultaten der Thiersch'schen Untersuchungen gebracht worden sind, nicht durch Gründe und Beweise erhärtet warden. Protocolle wie sie Fischer giebt z. B. das erste S. 18: "Die Wunde bildet im mikroskopischen Präparat eine leicht klaffende Spalte, welche grösstenthoils gefüllt ist mit Leukocyten, doch bemerkt man zwischen denselben deutlich gestreckt verlaufende Fibrinfäden." Solche Protocolle, meine ich, können in Anbebracht der Streitigkeit über das Wesen und die Herkunft der Zellen und der Verklebungsmasse nur als persönliche Anschauung eine gewisse Bedeutung beanspruchen. 
Die Annahme, dass alle die polynucleären Elemente Leukocyten sind, war erst dann begründet, wenn nachgewiesen wurde, dass diese Formen auf keine andere Weise als durch Auswanderung aus dem Blutstrom entstehen könnten. Dieser Beweis ist aber noch nirgends erbracht. Wohl aber haben zu allen Zeiten einige Forscher sich vor diesem fehlerhaften Schlusse gehütet und entgegen der Emigrations-Doctrin Bilder und Gründe veröffentlicht, die zwar die begeisterten Anhänger der Cohnheim'schen Lehre nicht überzeugen konnten, aber doch zum mindesten auf die Unsicherheit und Mangelhaftigkeit im Fundamente des Lehrgebäudes von der ausschlaggebenden Bedeutung der Leukocyten aufmerksam machen mussten.

Wir wollen also an die Untersuchung herangehen, nicht in der Voreingenommenheit, jeder u- oder s- oder kleeblattförmige Kern gehört einem Leukocyten an, sondern wir wollen bei jedem einzelnen Dinge, auch den mehrkernigen Elementen prüfen: „Kann oder muss dieser Kern nach Grösse, Form, Lage u. s. w. für einen Lenkocyten gehalten werden?" Nur auf diese Weise wird man zu einem verlässlichen Urtheil gelangen können.

Der dritte Punkt, den wir bei unseren Untersuchungen ganz besonders beachten wollen, ist die Frage, welche Rolle die Grundsubstanz bei den Gewebsveränderungen spielt. Dieselbe ist in sämmtlichen oben angeführten Arbeiten völlig unberücksichtigt geblieben. Man hat sich gewöhnt, die Grundsubstanz zu übersehen, hat sich mit einer allgemeinen Wendung, wie z. B. „die Grundsubstanz wird eingeschmolzen“ abgefunden. Beobachtungen über den Modus der „Einschmelzung" liegen aber nur bei Stricker und Heitzmann vor. Heitzmann ${ }^{1}$ ) denlst sich alle Gewebe durchzogen von einem feinverzweigten Kanalsystem von Protoplasma (Bioplassonnetz) in dessen Maschen hinein die todte Grundsubstanz abgeschieden ist. An den Knotenpunkten liegen die Zellen und Kerne (Plastide). Bei Reizungen kann an jeder Stelle des Netzes ein Protoplasmakörnchen zum Kern auswachsen und dabei die todte Grundsubstanz der Nachbarschaft resorbiren.

1) C. Heitzmann, Mikroskopische Morphologie des Thierkörpers im gesunden und kranken Zustande. Wien 1883. 
Stricker ${ }^{1}$ ), der seine Untersuchungen uber Wundheilung an Froschcornea und Trachealknorpel der Kaninchen gemacht bat, sagt in den davon handelnden Kapiteln seines Lebrbuches nichts von einer Betheiligung der Grundsubstanz. In der letzten seiner Vorlesungen handelt er im Allgemeinen von der Grundsubstanz der Cornea und schliesst sich hier der Ansicht Heitzmann's an, dass an jeder Stelle der Intercellularsubstanz Zellen entstehen könnten. Somit war das Hauptprincip, dass die Grundsubstanz lebe, zum Ausdruck gebracht, und Stricker wünscht das Princip auf alle Gewebe und Yorgänge übertragen. Die Uebertragung des Princips ist aber kein Beweis; dies seheint der Grund, dass in dem ausfübrlichen Capitel über Histologie der Wundheilung in Billroth.Winiwarter's Chirurgie 1893 eine Zellenbildung aus Grundsubstanz nicht erwähnt wird, obgleich auf Stricker's Autorität Bezug genommen ist. Wir halten für durchaus nothwendig, die Entzindung an entzündeten Geweben, die Heilung an heilenden und die Heilung der menschlichen Haut auch wirklich an menschlicher Haut zu studiren.

Dieses Princip der directen Untersuchung krankhafter Vorgänge liegt einer Reihe von Arbeiten zu Grunde, welche in den letzten Jahren aus dem Greifswalder pathologischen Institute ${ }^{2}$ )

9) S. Stricker, Vorlesungen öber allgemeine und experimentelle Patho. logie. Wien 1883.

3) Grawitz, Ueber die schlumwernden Zellen des Bindegewebes und ibr Verhalten bei progressiven Ernährungsstörungen. Dieses Archiv. Bd. 127. - W. Viering, Experimentelle Untersuchungen über die Regeneration des Sebnengewebes. Dieses Archiv. Ba. 125. S. 252. - Il. Schmidt, Schlummernde Zellen im normalen und pathologisch veränderten Fettgewebe. Dieses Archiv. Bd. 128. - A. Kruse, Uober Entwickelung, Bau und pathologische Veränderungen des Hornhautgewebes. Dieses Arehiv. Bd. 128. - R. Krösing, Ueber Rückbildung und Entwiekelung der quergestreiften Muskelfasern. Dieses Archiv. Bd.128. C. W. Schleiffarth, Ueber die Entzündung der serösen Organbedeckungen und der Gebirnhäute. Dieses Archiv. Bd. 129. - C. Kickhefel, Zur Histologie und zur systematischen Stellung der schleimigen oder gallertigen Gewebe des Menschen. Dieses Archiv. Bd. 129. A. Tenderich, Untersuchungen äber genetische und biologische Veriältnisse der Grundsubstanz des Hyalinknorpels. Dieses Archiv. Bd. 131. - Paul Grawitz, Atlas der pathologischen Gewebelehre. Berlin, Verlag bei Richard Schootz, 1892. 
zumeist in diesem Archive veröffentlicht worden sind, und die insgesammt dem Verhalten der Grundsubstanz bei den Gewebsveränderungen eine bis dahin völlig ungewohnte Beachtung und Aufmerksamkeit gewidmet haben.

In dem gleichen Sinpe bin ich in der vorliegenden Abhandlung bestrebt gewesen, die oft recht verwickelten Bilder, welche sich von den ersten Anfängen einer Verletzung bis zur vollständigen Heilung in der menschlichen Haut abspielen, unter Berücksichtigung aller Umstände, unter denen sie entstanden sind, direct zu interpretiren, ohne zur Erklärung andere Bilder herbeizuziehen, welche bei Thierversuchen unter Bedingungen gewonnen sind, deren Uebereinstimmung mit der Wundheilung mir sehr fraglich erscheint.

Soviel diene zur Orientirung über den Stand der Frage; Genaueres und Einzelheiten aus der hierher gehörenden Literatur enthält meine Inaug.-Dissert.: "Ueber die Heilungsvorgänge an Schnittwunden der Haut" (Greifswald 1892). Nach diesen einleitenden Bemerkungen komme ich jetzt $z a$ der Beschreibung und Erklärung der von mir gefundenen Bilder.

Bei meiner Untersuchung konnte ich mich ausschliesslich lebenswarm fixirter, auf der hiesigen chirurgischen Klinik gewonnener Gewebsstücke aus menschlicher Haut bedienen. Und zwar wurden sie in der Haut des Armes oder der Brust meiner Freunde oder in der Haut meines eigenen Armes zum Theil von Herrn Professor Helferich selbst angelegt und exstirpirt.

Dabei wurde unter peinlichster Beobachtung strengster Asepsis verfahren. Die Wunden wurden mit Seide genäht nnd nach verschieden langer Heilungsdauer mit ihrer Umgebung herausgeschnitten. Die Gewebsstücke sind dann in der zweckentsprechenden Grösse sofort in ein bereit stehendes Fläschchen Flemming'scher Lösung (einzelne auch in absolutem Alkohol oder concentrirter Sublimatlösung) fixirt, gründlich gespült, in Alkohol nachgehärtet und in Paraffin eingebettet geschnitten worden. Die Schnitte wurden in sehr verschiedener Weise ge(ärbt $\left.{ }^{1}\right)$.

1) Saffranin mit und ohne Pikrinsäurezusatz bei der Entfärbung. Carbolfuchsin-Pikrinsäurealkohol. Weigert'sche Fibrinfärbung. Anilinwassergentianaviolett und Entfärbung in Alkohol, theilweise mit Gegen- 


\section{Zur Untersuchung dienten 12 verschiedene Wundpräparate und zwar}

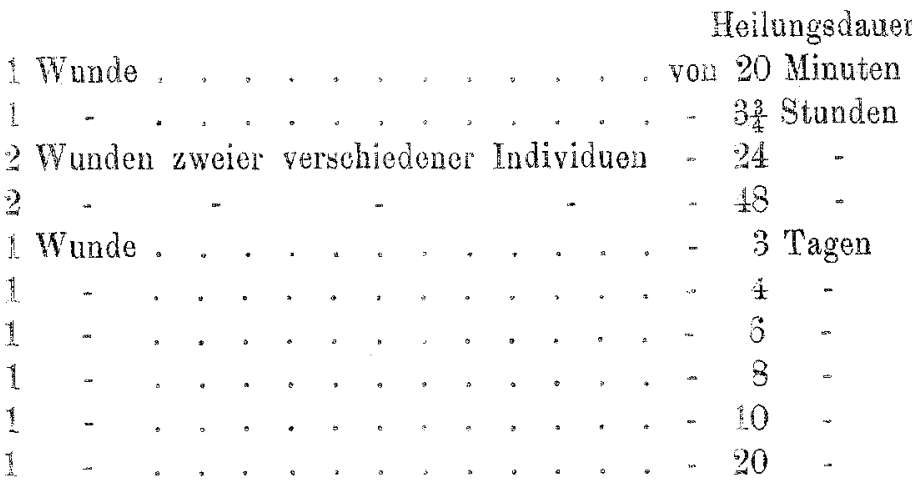

Der Befund in den ainghen Fälen war der rolgende.

20 Minuten.

Die sengrecht dureh die Haut und im rechten Winkel zun Wundverlaus geschnittenen Präparate zeigen übereinstimmend den einen Wundrand umgelegt, so dass die ganze Epithellage 1-2 $\mathrm{mm}$ weit in die Wunde hineinragt, während ihr am anderen Wundrande durchschnittene Cutis anliegt.

1) Die Präparate aus der Mitte der Wunde enthalten einen Wundspalt, in welchem rotbe Blutkörperchen, einzelne kleine Zellen von Leukocytentypus und Fettropfen liegen. Nirgends ist in dem Gerinnsel ein Netzwerk zu sehen, welches nach Saffraninfärbung noch rothe Fasern enthielte; weiter in der Tiefe sieht man die breiten Faserbündel beiderseits neben dem Wundspalte vielfach aus einander gedrängt, so dass zahlreiche Nebenspalten von kurzem oder längerem Verlaufe sichtbar sind, welche rothe Blutkörperchen entbalten. Wo eine Talgdrüse getroffen ist, sieht man auf mebrere Millimeter weit feinste schwarz gefärbte Fetttröpfchen in den Spalten liegen. Die Grundsubstanz zeigi bis an den Wundspalt heran dasselbe Lichtbrechungsund Färbungsvermögen wie die entfernter liegenden Abschnitte. Man unterscheldet breite, fast bomogene oder fibrilläre Bündel von hellgelber, bei Carbolfuchsinfärbung hellgrauer oder violetter (leimgebender) Grundsubstanz, eine grosso Menge zwischen ihren Bündeln oder auch durch sie hindurchziehender, schwarzblauer, bei reiner Saffraninfärbung rother oder blaugrauer Q eastischer Fasern. Im Yerlaufe der Blutgetässe und Dräsen färben sich veichlichere ovale Kernmembranen mit 1-2 Kernkörperchen, die dicken $\mathrm{F}_{\mathrm{a}}$ serbündel entbalten in weiten Abständen von einander längliche oder rundliche Kerne, die auch zuweilen besonders an den Knotenpunkten von drei dicken Bündeln eine dreieckige Gestalt annehmen und zu webreren neben

färbung durch Eosin oder Pikrinsảure. Methylenblau und Entfärbung wureh Alkohol. 
einander liegen, aber nur ausnahmsweise eine Spur von körniger Zellsubstanz erkennen lassen.

In dem Verlauf der elastischen Fasern oder diesen eng anliegend färben sich ebenfalls kleinere und grössere Kerne, an welchen selbst bei stärkster Braunfärbung keine Zellplatte zu bemerken ist (Fig. 9, k). Die grosse Mehrzahl dieser Kerne entspricht dem, was Köllikerl) in seiner Gewebslehre (1863) S. 79 als Faserzellen abbildet; im rubenden Zustande sind nur sehr wenige wirkliche $Z$ ellen in der Grundsubstanz vorbanden, die Mehrzahl der sogenannten fixen Gewebszellen sind Faserbündel mit erhaltenen Kernen.

Unter den innerbalb der blansehwarzen elastischen Fasern liegenden Chromatinklümpchen sind viele, welche lsaum als feinste Pünktchen oder Striche wahrnebmbar sind, und keiner auch noch so kleinen Kernform wirklicher Zellen entsprechen.

2) Die Schnitte, welche nicht der Mitte, sondern nahe den Wundwinkeln entnommen sind, zeigen nur einen minimalen Spalt, so weit das Epithel des einen Randes hinabreicht; von hier ab deutet nur ein intensir rotber, selbst bei Entfärbung mit Pikrinsäure-Alkohol nicht entfärbter Streifen die Richtung der Trennung an, es ist völlige Verklebung eingetreten. Auch oben, an den Epithelsanm anstossend, ist eine feine rothe Grenzlinie in dem durchschnittenen Cutisgewebe sichtbar. Von dem rothen Streifen setzen sich mebrfach feinste rothe Ausläufer in die benachbarten Bündel fort, ähnlich wie in Fig. $8 \mathrm{f}$. Diese Ausläufer baben an den Knotenpunkten kleinste dreieckige Chromatinanschwellungen (Fig. 3 and 4), im Verlaufe des dickeren Streifens finden sich bellere ovale Läcken, in denen ebenfalls Gebilde, wie kleine Nucleoli eingelagert sind. Ueberall, wo das Messer die Gewebe getrennt hat, ist der rothe Saum sichtbar; da wo sich von dem Saum zarte rothe Fasern (Fig. $8 \mathrm{f}$ ) in die Spalten benachbarter leimgebender Bündel abzweigen, entsteht der Anfang eines rothen Netzwerkes, dessen rothe Fasern bald in ungefärbte Fortsätze übergeben. In den Maschen des Netzes liegt normal gefărbte, oder mit Blutfarbstoff getränkte Grundsubstanz wie in Fig. 8 bei G.

Die Gefässe in der näheren und weiteren Umgebung der Wunde zeigen ein durebaus normales Ausseben, in Sonderbeit ist von einer Anbäufung von Leukocyten in oder um die Gefässe absolut nichts zu bemerken.

Epikrise. Die Wundränder sind verklebt durch eine rothe Substanz, von der aus Fäden in die Grundsubstanz sich fortsetzen. Wo im Wundspalte geronnenes Blut liegt, fehlt die rothe Substanz innerhalb des Gerinnsels gänzlich, dagegen ist sie im Grenzsaum des Gewebes sichtbar. Wenn also die verklebende Substanz Fibrin wäre, so müsste man annehmen, dass die Gerinnung nach 20 Minuten erst hart am Gewebe und in den angrenzenden Gewebsspalten aber noch nicht inmitten des er-

1) Kölliker, Handbuch der Gewebslehre des Mensehen. IV. Ausgabe. 1863. 
gossenen Blutes eingerreten seil. Dass dies nicht zutrifft ergiebt sich daraus, dass wir in zahlreichen Schnitten einer 6 Tage atten Wunde and in noch spateren Stadien immer noch Blutcoagula obne ein Maschenwerk antreffen, während das rothe Netzwerk, welches bei 20 Minuten nur hin nd wieder ein wirkliches Netz zeigte, von Stunde an immer weiter und weiter in dies Nachbargewebe vordringt.

Wir haben oben gesagt, dass nur ein ganz kleiner Theil der normaleweise im Bindegewebe vorhandenen Kerne eine Spur von Zelleib erkennen lasse. Wir nehmen nehmlich auf eine Reihe eigner Beobachtungen gestütat mit Schwann, M. Schultze, Stricker u. A. an, dass die fibrilläre Zwischensubstanz durch oine directe Unwandlung der Zellen selbst, nicht etwa durch sine Abscheidung der Zellen entstehe. Die Grundsubstanz ist nicht ein todtes Ausscheidungsprodukt sondern ein an allen Tebensvorgängen activ thellnehmendes Stadium, eine besondere Erscheinungsform des lebenden Protoplasmas. Wenn nuu auch die Zwischensubstanz in dem derben Cutisgewebe den Lobenszustand darstellt, der für die ungeheure Mehrzahl der das Gewebe aufbauenden Zellen die Norm ist, so kommt os doch bei der einen oder anderen derselben nicht zu völliger Ưmwandlung, sondern dieselben bleiben als nsogenannte Bindegewebskörperchen" in irgend einem Uebergangsstadium erhalten. So sind die mehr oder minder grossen Kerne and Zellen in den Bindegewebsbündeln zu erkiären, so die den elastischen Fasern anliegenden oder eivgelagerten Chromatinfiguren.

\section{Stunden。}

Die Präparate lassen bei schwacher Vergrösserung einen schmalen, in tensiv roth gefärbten, zum Theil etwas klaffenden Streifen erkennen, welcher der ursprünglichen Wunde entspricht. Dieser Streifen ist am oberen Rande trichterartig erweitert and hier von dem beiderseitig etwas eingebogenen Epithelsaum begrenzt. Das in geringer Menge ergossene Blut ist ohne Netzwerk, aber von einem solchen begreazt. Der Streifen ist niebt überall von gleicher Dicke, sondern zeigt viele Anschwellungen und Fortsätze, welche in die die derben Gewebsbündel trennenden Spalten sich fortsetzen. Nach unten zu verläuft dieser Streifen auch in erweiterte Saftkanälehen. Das Cutisgewebe enthält hart am Wundspalt eine grosse Zahl kleiner Chromatingebilde, welche vom Wundspalt her ziemlich weit in Verlauf der benachbarten Saftlücken und Gewebsspalten zo verfolgen sind. In dem loseren Bindegewebe um Gefässe, Nerpen und Drüsen sind neben einzelnen Gewebszellen kleinste und 
grössere Kerngebilde in engen Abständen und mebrfachen Reiben sichtbar, aber nirgends so reichlich als hart an der Verklebungsstelle.

Bei der Untersuchung mit stärlkster Vergrösserung löst sich der vorher einfach intensiv roth erscheinende Streifen in ein stark mit Saffranin imbibirtes, gefärbtes Maschenwerk auf. Die Balken des Netzwerkes sind nicht von gleicher Dicke, vielmehr sehen wir tiberall einige besonders starke Linien an den Wundrändern entlang ziehen. An Stellen, wo die Verklebungsmasse etwas breiter ist, befinden sich noch mehrere ähnlich starke, ungefäbr in derselben Richtung verlaufende Balken, die von eben solchen gekreuzt werden (vgl. Fig. 8). Von diesen stärkeren zweigen sich schwächere, von diesen noch feinere, weniger intensiv gefärbte Linien $a b$, die communiciren und in dem grobmaschigen ein feinere Naschen enthaltendes Netzwerk bilden. Dieses Netzwerk pflanzt sich auch in die benachbarten Gewebstheile fort und zwar derart, dass diese roth gefärbten Linien Felder umschliessen, die unzweifelhaft aus unveränderter Intercellularsubstanz des Bindegewebes bestehen. Die rothen Linien werden mit der Entfernung von der Wunde beller und gehen allmählich in ganz helle, die Bindegewebsbündel trennende Linien ïber, die nur hier und da feinste rothe Anschwellungen tragen (Fig. 3 und 4).

Doch bleiben wir zunächst noch bei dem Wundspalt selbst und betrachten uns genauer, was zwischen den oben beschriebenen, netzförmig angeordneten rothen Linien liegt.

Erstens haben viele der feinen rothen Linien spindlige oder rundliche, intensiv roth gefärbte Anschwellungen (Fig. 3); oft sehen bei 500facher Vergrösserung die rothen Figuren ans, als wenn es anastomosirende Sternzellen, bei 50 facher Vergrösserung betrachtet, wären. In den kleinen, bei Pikrinsäurefärbung hellgelben Feldern innerhalb der rothen Linien sieht man intensir rothe, runde oder ovale Körnchen, längliche oder dreieckige Gebilde vom Färbungsvermögen der Nucleoli liegen (Fig. $3 \mathrm{~N}$ ), welche sich in keiner der oben erwäbnten Arbeiten über Wundheilung beschrieben finden. Wo $z$ wei oder drei dieser kleinen Chromatinhäufchen neben einander liegen (Fig. 3 N, Fig. 1 N), gleichen die Bilder ausserordentlich Leukocytenkernen. Von diesen Kerngebilden sind eine Reihe anderer verschieden, die (wie dies Fig. $3 \mathrm{E}, 8 \mathrm{E}, 4 \mathrm{E}$ veranschaulicht) bis zu den kleinsteu Formen hin den Typus der Endothelkerne zeigen. Sie liegen, wie jene anderen auch, im Verlaufe oder an den Knotenpunkten der rothen Fäserehen oder auch in der gelb gefärbten Grundsubstanz inmitten der Maschen und lassen auch in den kleinsten Formen eine mehr regelmässige ovale Gestalt und eine intensiv gefärbte Kernimembran erkennen. Wir müssen uns hier die Entsagung auferlegen, diesen mit den Kernfärbemitteln sich stark färbenden, also kernähnlichen Gebilden sogleich einen Zellenleib zuzusprechen; einen solchen kann man besonders bei den kleinsten Formen auf keine Weise zur Anschanung bringen. Wir dürfen diese also weder als Leukocyten noch ais permanente Gewebszellen ansprechen, sondern müssen uns darauf beschränben, ihrer Lage nach zu sagen, dass der eine Theil von ihnen mit elastischen Fasern, ein anderer mit der gelben leimgebenden Grundsubstanz zusammen- 


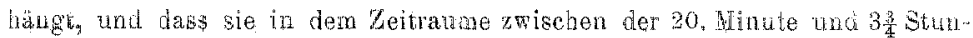
den nach der Verletzong färbbar und sichtbar geworden sind. Wir müssen ferner hervorheben, dass diese Formen um so kleiner und unvollkoromener werden, je weiter wir uns von den Stellen mit hochgradiger sogenannter "kieinzelliger Infiltration“ in das mebr normale kernarme Nachbargewebe begeben. Verschiebt man das Prapparat in dieser Weise, so kann man sowohl die mebrkernigen lenkocytenartigen, als die endothelartigen Gebilde in allen Grössen von der ausgebildeten Zelle bis binab zu dem kleinsten mit den stärksten Vergrözserungen kaum wahrnehmbaren Pünktehen antreffen.

Wofür soll man nun diese Dinge halten? Anfrecht, Fiseher, Nikiforoff haben sich mit allen diesen Formen summarisch abgefunden, indem sie alles für Leukocyten erklärten. Diese Dentung der beschriebenen Kernforroen als bierher gewanderter Lenkocyten würde voraussetzen, dass die in der Nähe der Wunde angetroffenen fürbbaren Gebilde die auf der Wanderung begriffenen farblosen Blutzellen sind. Selno dae ganz ausserordentliche Grössenverschiedenheit der beschriebenen Gebilde macht aher diese Deutung tür den grossen Theil der Elernente hinfallig. Da wir es bei der flinwanderung von Leuksocyten mit fertigen, aus dem Blute stammenden Zellen $z u$ thun haben, deren Grösse nur innerhalb sehr enger Grenzen schwankt, so unuss man also in erster Linie alle die ganz kleinen Formen pon der Klasse der Lankocyten ausnehmen, die jodesmal in der Peripherio von einem Haufen keiner Rundzellen angetroffen werden.

Aber auch eine sehr grosse Zahl der den farblosen Blutzellen gleichenden Gebilde erweist sich bei genauer Prüfung als andersartige Elemente. Dies sind zunächst diejenigen, die auch bei intensivster Gegenfärbung, bei stärkster Vergrösserung und in allerdünnsten Schnitten nicht eine Spur von Rellsubstanz erkennen lassen, sondern (wie dies Fig. 1, 3, 4, 7, 9 zeigt), entweder inmitten der unveränderten leimgebenden Faserbündel oder innerhalb der elastischen Fasern liegen. Fs bleibt nun somit nur noeh ein kleiner

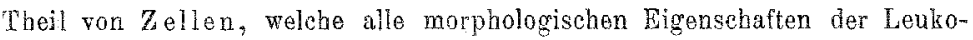
syten haben. Und auch bei dieser muss man den weitaus grössten Theil ron der Dentung anf der Wanderung begriffener farbloser Zellen ausschliessen.

Sobald man nehrolich ganz dünne Schnitte betrachtet, in welchen durch ungleiche Alkoholschrumpfung die Gewebsspalten im Verlaufe der Bindegewebsbündel sich erweitert baben, sieht man, dass die Chromatinhäufchen, sofern sie wirklich innerbalb einer Spalte liegen, den blauen elastischen Fasern angehören (Fig. 1, 4, 7,9), und dass die übrigen nicht frei in den Spalten, sondern innerhalb der den Spait begrenzenden gelben Grundsubstanz liegen.

Es sprechen also gegen die Abstammung der Gebilde aus dem Blute 1) die ansserordentlich kleinen (hromatingebilde (Fig. 3 N), 2) die ausserardentlich vielen Abstufungen dieser kleinsten Nucleolen zu grösseren Kernformen, 3) der Mangel abgrenzbarer Zellsubstanz, 4) die Lage der Gebilde im Verlauf der elastischen oder der leimgebenden Faserbündel, 5) das Fehlen derselben in den Gewebsspalten. 
Für die Leukocytennatur spricht nur der Umstand, dass man im losen Bindegewebe um Blutgefässe und in denselben oft die gleichen Kernformen liegen siebt. Aber auch um die Nervenbündel, uw die Talg- und Schweissdrüsen finden sich nach $3 \frac{3}{4}$ Stunden concentrisch angeordnet dieselben bald multinucleären, bald einfachen, bläschenförmigen, ovalen, länglichen Kerne (vom Endotbeltypus) in der Art, dass immer die grösseren nahe der Drüse oder dem Blutgefässe liegen, während die entfernter gelegenen sich bis zu den Kleinsten Kugel- oder Stäbchenformen abstufen. Grosse Zellen vow Typus der Gewebszellen, wie in Fig. 7, 8, 9, sind nach $3 \frac{3}{4}$ Strnden im derben Bindegewebe nirgends in der Nähe der Wunde sichtbar. Mitosen oder schön fixirte Kerngerüste haben wir nur in den Epithelzellen getroffen.

Epikrise. Die Präparate lehren, dass das rothe Fasernetz nicht in den eigentlichen mit Blut erfüllten Wundspalt, sondern in das benachbarte Gewebe vorgerückt ist. Die Erklärung dieser Substanz, ihr Werth and Zustandekommen ist noch völlig ungewiss. Thiersch hielt sie für eigenthümlich gequollenes Gewebe, während alle späteren Forscher diese Verklebungsmasse für ein in den Wundspalt ergossenes Exsudat erklärten, das dem Fibrin in seinem chemischen und physikalischen Verhalten völlig gleichwerthig sei. Demgegenüber müssen wir nun hervorbeben, dass das Aussehen dieses Fasernetzes, sich von dem des Fibrins erheblich unterscheidet. Wir finden sonst im Fibrin ein wirres regelloses Durcheinander von Fasern und Fäserchen, die sich mit den Kernfarbstoffen nicht tingiren. Hier dagegen sehen wir grosse von dicken stark roth (mit Saffraniu) gefärbten Balken umgebene Felder, die durch ziemlich regelmässig von den Balken abgehende feinere Aeste in kleinere Abschnitte getheilt werden. Wenn diese Masse also überhaupt Fibrin ist, so ist sie jedenfalls eine besondere Art desselben.

Wenn dasselbe nun ein Exsudat ist, so kann entweder die in den Wundspalt ausgeschiedene Gewebsflüssigkeit oder Lymphe zusammen mit dem dort schon vorhandenen Blut als gemeinsames Gerinnungsprodukt dies Fasernetz liefern (so Gussenbauer) oder das Blut allein kann das Fibrin bilden, oder endlich die Gewebsflüssigkeit allein kann dies Gerinnungsprodukt bilden.

Gegen die ersten beiden Möglichkeiten spicht das Aussehen der Blutcoagula im Wundspalt. Wäre das Blut irgendwie erheblich an der Bildung betheiligt, so müssten wir im Blutge- 
rinnsel am allermeisten sein Gerinnugsprodukt vorinden. W11 sahen aber, dass dies nicht der Fall ist, dass grössere Hämorrhagien, die wir z. B. in den Rraparaten yom 6. Tage besonders reichlich antrafen, absolut frei sind von einem ähnlichen Maschenwerk. Nur die Peripherien derselben, soweit sie in den Gewebsspalten liegen, weisen an einzelnen Stellen ein derartiges Maschenwerl: auf.

So bliebe denn noch die dritte Möglichkeit, dass der Gewebssaft allein dasselbe liefert. Diese Annahme ist aber ganz unvereinbar mit der Thatsache, dass die am Ausgange des Wundspaltes auf der Epitheldecke gelegenen Kernmassen ganz regellos ohne irgend oine Andeutung eines solchen Maschenwerks sind. Ist die Verklebungsmasse wirklich ein Exsudat, dann ist der an der Obertäche der Wunde gelegene Kernhauten oben nur dor aus der Wunde hervorgetretene Theil des Exsudates und muss als solcher füglich dieselbe Zusammensetzung zeigen wie der in der Wunde gelegene Theil. Auch der einzig noch mögliche Einwand, der zur Erklärung dieses in die Augen fallenden Widerspruches herangezogen werden kann, dass nehmlich der oberhalb der Epitheldecke belegene Theil etwa eintrockne oder sich sonstwie verändere, ist nicht stichhaltig. Denn in denjenigen Präparaten, in denen der eine Epithelsaum weit in den Wundspalt hineingebogen ist, lässt die diesem Saum aufliegende "Exsudatschicht" nicht die geringste Spur dieses Fasernetzes erkennen, selbst nicht in der Tiefe der Wunde (also etwa 2 mma unter dem oberen Rand), während hingegen die dem gegenüberliegenden Wundrand (also dem durchschnittenen Corium) zugebörige Partie des vermeintlichen Exsudates bis an das Rete Malpighi hinauf dieselbe Struktur aufweist wie der Spalt in der Tiefe.

Hierdurch erscheint mir der Beweis erbracht, dass die Verklebungsmasse kein Exsudat ist. So bliebe deno die Möglichkeit mit Thiersch anzunohmen, dass dieses Maschenwerk eigenthümlich gequollenes Cutisgewebe selbst sei.

Gussenbauer ${ }^{1}$ hatte als Hauptgrund hiergegen angeführt, dass man Bindegewebskörper in dem. Maschenwerk nothwendiger Weise antreffen müsste, wenn anders dasselbe verändertes Bindegewebe sei, man träfe aber nur Wanderzellen. Dieser Grund

1) Siehe oben. 
ist nicht zutreffend, weil Gussenbauer den Begriff der Wanderzelle und farblosen Blutzelle viel zu weit fasste. Wir haben unsere abweichende Ansicht schon oben kurz gestreift and kommen daranf unten noch einmal wieder ausführlich zurück. Hier genüge die Bemerkung, dass wir den Einwand Gussenbauer's als absolut beweisend nur dann ansehen wärden, wenn irgendwo nachgewiesen wäre, dass die Gewebszelle unter keiner Bedingung eine Zerklumpung ihres Chromatins zu den charakteristischen mehrkernigen Formen, wie wir sie boi den Leukocyten antreffen, erfahren könnte.

Wir können aber einige positiv für die Ansicht von Thiers ch sprechende Beobachtungen anführen.

Wir haben schon oben in dem Protocolle gesagt, dass in den ersten Stunden noch mitten in dem Maschenwerk grosse Bündel relativ unveränderter Grundsubstanz vorhanden sind. Auch Nikiforoff sagt:

1) In dem Grenzgebiet (zwischen Fibrin und Bindegewebe) sind die fibrinösen Massen weniger dicht und bilden ein zartes Fibrinnetz, in dem noch gut erhaltene Bindegewebsbündel erkennbar sind.

Die Erklärung dieser Thatsache bei Annahme eines Exsudates würde wohl sehr schwierig werden in Sonderheit, wenn man beachtet, dass in den späteren Stadien des Heilungsverlaufes keine derartigen Bindegewebsbündel mehr im eigentlichen Fibrinnetz sich vorfinden. Auch wäre bei dieser Annahme nicht verständlich, weshalb die fibrinösen Massen im Grenzgebiet weniger dicht sind und ein zarteres Netz bilden als die weiter entfernten Partien. Ich halte dafür, dass die fibrinoide Umwandlung des Gewebes aller Theile des Grenzbezirks in den angeführten Fällen noch nicht vollendet ist. Darum treffen wir noch erhaltene Grundsubstanz in dem Maschenwerk, darum sieht dies an der Grenze zum Bindegewebe noch zarter aus als in der Mitte des Wundspaltes.

Ein weiterer Beweis für die Entstehung der fibrinoiden Substanz aus dem Bindegewebe ist der Umstand, dass sich die Balken des Maschenwerks direct in die ungefärbten Trennungslinien zwischen den Bindegewebsbündeln fortsetzen (vergl. Fig. 8). Denn man kann doch unmöglich annehmen, dass die ungefärbten Linien, die direct in die rothen Leisten übergehen, den Theil 
des "Fibrinexsudates" darstellen, der sich in den Gewebsspaiten befindet. Ein derartiges verschiedenes Verhalten desselven Exsudates gegen die Farbstoffe wäre absolut nnerklärlich.

Dass eine solche fibrinoide Umwandlung des Bindegewebes möglich ist, das zeigen die vielfach in der Nachbarschaft der Wunde im Gewebe vorhandenen Netzwerke, die ein oder mehrere Bindegewebsfelder ganz oder theilweloe einnehmen.

Auch ist Thiersch nicht der erste und einzige gewesen, der diesen Vorgang beschrieben hat. Schon Rokitansky') wies auf solche Veränderung des Bindegewebes bei Entzûndung der serösen Hänte hin. Virchow ${ }^{2}$ vertritt eine active Betheiligung des Bindegewebes bei der Bildung des Fibrins, indem er annimmt, dass das Fibrin nicht einfach ans dem Blute stammende an der Oberfläche gerinnende Flüssigkeit sei, sonderm dass es burch eine Absonderung der Zellen gebildet wird. Buhl ${ }^{3}$ ) gelang es sogar darch Injectionspräparate noch Gefíssschlingen in dem sogenannten Fibriubelage nachzuweisen.

Im Jabre 1880 hat dann E. Neumann ${ }^{*}$ ) unter Anwendung der neuen Pikrocarminfärbung gefunden und beschrieben, dass die vormeintiche Fibrinauflagerung bei Entzündung der serösen Häute, sowie der Gelenkkapseln und Sehnenschoiden aus einer "fibrinoiden Degeneration" des Gewebes selbst entstehe.

In veuester Zeit hat anch Schleiffarth ${ }^{5}$ ) ans dem pathologischen Institut zu Greifswald eine Reihe von Untersuchugen veröffentlicht, die ihn betreff dieses Gegenstandes zu demselben Resultat geführt haben, nachdem schon Schuchardt im Jahre 1890 die Umbildung des Bindegewebes zu Fibrin gelegentlich einer Untersuchung über die Hygrome hatte constatiren können.

Ich selbst konnte mir unter Anderem gelegentlich der Untersuohung eines exulcerirten Iymphangioms der Zunge absolute

1) Rokitansky, Lebrbrich der pathol. Anatomie. 3. Aufl. 1855. Bd. 1.

2) Virchow, Gesammelte Abhandlungen. 1856. S. $13 \mathrm{~B}$.

3) Buhl, Ueber das Faserstoffexsudat. Sitzungsber. d. Kgl. Bayer. Alkad. d. Wissensch. 1863. Bd. II.

1) K. Neumann, Die Pikrocarminfärbung und ihre Anwendung auf die Entzündungslehre. Arch. f. mikroskop. Anat. 1880. Bd, 18. S. 130.

3) C. W. Schleiffartb, Ueber die Entzündung der serösen Organbedeckungen und der Gehirahäute. Dieses Archiv. 1892. Bd. 129.

G) Schuchardt, Dieses Archiv. Bd.121. S. 305. 
Gewissheit darüber verschaffen, dass wirklich eine fibrinähnliche Umwandlung des Gewebes vorkommt. Eine Vene, die mit einer Hälfte ihrer Circumferenz über den Geschwürsgrund hervorragte und prall mit Blut erfüllt war, zeigte $W$ andungen, die nicht aus Bindegewebe oder Muskelfasern, sondern aus einem fibrinösen Maschonwerk bestanden.

Nach allem diesem besteht für mich kein $Z$ weifel, dass auch hier bei der Heilung aseptisch genähter Hautwunden die Ränder derselben fibrinartig aufquellen und, falls nicht etwa ein Blutcoagulum zwischen ihnen liegt, direct verkleben.

Neben der Ausbildung dieses fibrinoiden Maschenwerkes zeigen die Präparate den ersten Anfang einer „kleinzelligen Infiltration". Hierbei handelt es sich aber zum allergeringsten Theile nur um wirkliche Zellen (vergrösserte Gewebszellen und Leukocytenformen), in den allermeisten Fällen finden sich unfertige kleine Chromatingebilde in der verschiedensten Grösse in den elastischen Fasern und der leimgebenden Substanz.

Ueber den Werth der Zellen und das Zustandekommen der "kleinzelligen Infiltration" folgt ein eingehenderer Boricht nach Beschreibung der Präparate, die

\section{Stunden}

nach Anlegung der Wunde gewonnen worden sind.

Zur Untersuchung sind Hautstücke von zwei Personen und verschiedenen Körperstellen verwandt worden; einzelne davon sind nicht in Flemming'scher Lösung, sondern in Alkohol fixirt worden.

Das rothe Fasernetz ist am besten an solchen Schnitten zu beurtheilen, bei welchen in der Wundspalte Bluteoagula liegen. Man sieht alsdann oft auf lange Strecken einen hellgrauen Streifen des Gerinnsels, welcher keine Fasern enthält, beiderseits aber von einem zierlichen Gitterwerk begrenzt wird, welches in seinen Maschen Kernbildungen, Zellen oder gelbe Grundsubstanz zeigt. Die rothen Fäserchen, welche in der Verklebungsschicht nach 20 Minuten erst als schmaler Streifen mit feinsten Ausläufern angedeutet waren, und welche nach $3 \frac{3}{4}$ Stunden ein Reticulum mit Chromatinansehwellungen und kleinsten Kernformationen in den Maschen bildeten, sind jetzt zu einem Maschenwerk geworden, welches nach Fischerl) "neben

1) Otto Fischer, Experimentelle Untersuchungen über die Heilung von Schnittwunden der Haut. Inaug.-Dissert. Tübingen 1888.

Da in dem Netzwerke und oft beerdweise in der Nähe des Schnittes oder der Nahtstellen ganze Gruppen sternförmiger, grosser, granulirter 
2ablreighen eingewanderten mehrikerniggn Leukoegten auch acht wele en kernige Leukocyten und Hibroblasten" entbält. Bemerkenswerth ise, dass in dem Blutgerinnsel nur da etwas ron mebriernigen zellen zu seben ist, wo wit dem Blute zugleich in Wundspalt imbibirte, aber doel färbbare Grundsubstanz zu erkennen ist, wăhrend die Wundränder in diehten Reiben und Craufen "von Leulocyten infiltrirt sind".

Um die Herkunft der verschiedenen Zellen zu beurtheilen, muss wan sich wiedar der weiteren Ungebung der. Wunde zuwenden.

1) Auf Fig. 4 siebt man ein System blauschwarzer elastischer Fasern, deren Substanz nur in den dinneren Fasern noch homogen ist, während an Len dieteren Sielien, welche in ruhendem Zustande den drei parallelen Fasern der Fig. 1 entsprechen dürften, die blauschwarze Substanz hellere Lülicen und dunklere Klumpen unterscheiden lässt, in welchen in ganz regelmässigen Abständen S-förmige oder Lleeblattähnliche, intensiv rothe Partikelober ohne 2 ellbegrenzung liegen. In Fig. 7 siobt man ebenso rach $2 t$ Stunden in den Teriauf schwärzlicher elastischer Fasern länglich ovale kleinste Chromatinhäufchen, grössere ovale Kerne und endlich typische Endothelkarne mit feinstem Chronatingerüst eingeschaltet. Je grösser die ovalen Kerne sind, um so deutlicher ist an ihren Polen ein spindliger, tein granulirter, aber zunächst noch dunkelgrau - wie die elastischen Fasern - färbbarer Zellenleib zu unterscheiden, welcher sich unmittelbar in die blanschwarze Faser tortselzt. Da im rulenden Cutisgewebe im Verlaufe der elastischen Fasern nur ausnahmsweise wirkliche Zellen, yorwiegend kleine Chromatingebilde vorhanden sind, so kann nur ein kleiner Bruchtheil der in Fig. 7 dargestellten Zellen')

Zellen mit grossem Wndothelkern sichtbar sind, so tommt hier zum ersten Male für die Beurtheilung der Herkunit dieser "Fibroblasten" (iie mitotische Kerntheilung in Frage. Wir schicken also voran, dass wir hunderte von Präparaten, welche dem Zeitraume der ersten zwei lage angehören, mit grösster Sorgfalt unter Anweałung Aer Zeiss'schen apochromatischen Oelimmersion untersucbt haben, dass wir aber nur in den Epithelien Mitosen angetrofen baben. Da die Beschreibung der späteren Tage zeigen wird, dass wir rom dritten Tago ab in jedem Sebnitte schön fixirte Mitosen an Geîss- und Bindegewebszellen oft in grosser Anzabl gesehen haben, so bamn der Unterschied nicht in der Technils liegen.

1) Mikrophotographische Bilder dieser meiner Präparate sind in der dritten Lieferung des "Atlas der pathologischen Cexebelebre" von Grawitz enthalten. Die Photogramme beweisen in Folge shrer völligen objectivität mehr als lange Auseinandersetzungen sow'ohl das Vorbandensein der kleinsten Chromatinformen, wie auch den Zusammenhang der fibrinösen Verklebungsmasse mit der Grundsubstanz. Ein Vergleich dieser Photogramme würde eine treflicbe Ergünzung unserer Ausführungen und Abbildungen bilden. 
auf präexistirende Gewebszellen zurüekgeführt werden, die grosse Mehrzahl ist anch jetzt noch nicht als freie Zelle zu bezeichnen, da sie noch mit den Fasern zusammenhängen.

2) Neben den Saftlücken und neben den elastischen Fasern siebt man in der fibrillären oder mehr homogenen Grundsubstanz nicht allein kleinste Chromatinkörperchen, wie nach $3 \frac{3}{4}$ Stunden, sondern a) zahlreiche kleeblattähnliche, b) zahlreiche ovale, endothelartige Kerne wit mehr oder weniger gegen die Umgebung abgegrenztem Zellenleib. Hart an der Durchtrennungsstelle überwiegen die mehrkernigen (leukocytenartigen) Formen, etwas weiter entfernt davon die grossen anastomosirenden Zellen mit-ovalem Endothelkern und schönem Chromatingerüst in denselben. Die Entstehung dieser "Fibroblasten" veranschaulicbt Fig. 9, welche aus der Uebergangsstelle einer loseren Bindegewebsscheide einer Drüse zum derben Bindegewebe entnommen ist. Rechts sieht man einzelne Bruchstücke scbwärzlicher, elastischer Fasern mit Kernen, links Kerne verschiedener Grösse, welche zum Theil ganz direct von gelber Grundsubstanz umschlossen sind, zum Theil eine kleine, feinkörnige, helle Zone ringsum besitzen, die aussiebt als sei ein schmaler Saum der Grundsubstanz um den Kern herum eingeschmolzen, welche endlich eine deutliche körnige, färbbare Zellsubstanz erkennen lassen. Die Zellenleiber sind auch bei kleineren Formen sternförmig, mit Ausläufern versehen, welche einerseits die Zellen unter einander verbinden, und andererseits direct in die Grundsubstanz übergehen. Um die Kerne herum ist die Zellsubstanz oft so zart oder vielleicht flüssig, dass sie ganz homogen und selbst bei stärkster Behandlung mit Carbolfuchsin ganz farblos erscheint; oft fallen die Gebilde aus dünnsten Schnittstellen heraus, und die Grundsubstanz bekommt dadurch ein siebartig durchlöehertes Aussehen. Trotz der schönen Chromatinnetze innerbalb der Kerne sind keine Hitosen wabrzunehmen; anch unterscheidet sich die sternförmige Gestalt dieser Zellen von den später zu beschreibenden, durch Karyokinesis entstandenen, runden Gewebszellen.

Auch in der gelben Grundsubstanz liegen kleeblattähnliche "Leukocytenformen"; hart am Wundspalt zuweilen viele freie Gebilde, deren Form ganz und gar mit derjenigen von Lenkocyten übereinstimmt. An solchen Stellen, wo durch die Seidenfäden Umschnürung kleiner Blutgefässe stattgefunden hat, wo im Lumen kleiner Venen dichte Massen von Leukocyten liegen, wo in den Spalten des benachbarten Bindegewebes neben rothen Blutkörperchen zahlreiche mehrkernige kleine Elemente liegen, da scheint es uns unzweifelhaft, dass die letzteren aus dem Blute stammen. Schwieriger aber ist die Abstammung derjenigen Formen, welche, wie auf Fig. 2, 3, 4, 7 ersichtlich, a) im Verlaufe elastischer Fasern, b) in der fibrillären Grundsubstanz, c) in den Membranen der Fettkörper liegen und schon in den kleinsten Körnchenformen ans mehreren Chromatinklümpchen bestehen. Hiervon zu unterseheiden sind endlich noch wieder solche runden, scharf umrandeten, mit färbbarer Membran versehenen Körper, welche in Fig. 9 bei L abgebildet, an anderen Präparaten in reichlicher Menge beobachtet worden sind. Anch diese Formen sehen täuschend wie mehrkernige Leukocyten aus, 
allein es sind Berne mit verklumptem Chromatingentus, whe man sehr bostimmt an grossen anastomosirenden Sternzellen sehen kana, deren Zellleiber abwechselnd grosse Kerna wie in Fig. 9 and in anderen Exemplaren je einen solchen scbeinbaren Lenkocyten als Kem enthalten. Die Gronze des lenkooyterartigen Gebildes wird bier durch die Kornmembran gebildet.

Epikrise. Ein Vergleich der letztbeschriebenen Präparate mit den vorhergehenden zeigt, dass die verklebende fibrinoide Schicht an Breite zugenommen hat, nicht aber in die etwa vorhandenen Blutgerinnsel weiter eingedrungen ist. Auch fern von der Wunde im Gewebe gehen an vielem Stellen aus nicht ganz klaren Gründen solche Umwandlungen des Gewebes vor sich. In höherem Grade aber als die Fibrinbildung hat die "kleinzellige Infiltration" zugenommen. Wir finden zahlreiche Zellen und Kerne und kleinste Chromatinformen, zu deren Erklärung die althergebrachten und bekannten Theorien für das Lastandekommen der "kleinzeligen Infiltration" zunächst herangezogen werden sollen. Finden wir eine Vermehrung der Zellen im Ge. webe, so haben wir zur ihrer Erklärung

1) Die Emigrationstheorie,

2) Die Wanderung der Gewebszellen aus der Nachbarschaft,

3) Die mitotische und amitotische Theilung der Gewebszellea.

Mit diesen Mitteln können aber die in ungeheurer Anzahl nach der Verletzung im Gewebe sichtbaren Kleinen Chromatingebilde, wie wir sie in fast allen unseren Skizzen $z$. B. Fig. $3 \mathrm{~N}$, Fig. $7 \mathrm{~N}$, Fig. $9 \mathrm{~K}$ abgebildet haben und vie sie auf allen Photogrammen des oben citirten Atlas von Grawitz zu sehen sind, auf keine Weise gedeutet worden. Man könnte einwerfen, dass diese Chromatinhäufchen dadurch zu erklären sind, dass das Mikrotommesser nur gerade den Rand oder die Spitze eines Kerns abgetrennt habe. Dieser Einwand wïrde dann zutreffen, wenn wir diese Figuren nur an der Ober-oder Unterfläche der Schnitte fänden. Wir haben uns selostverständlich diesen Einwand selbst gemacht und speciell daraufhin die Präparate durchsucht. Dabei haben wir uns genugsam ubberzeugt, dass diese Gebilde in allen Ebenen der Schnitte in gleicher Weise anzutreffen sind, ihre Kleinbeit somit nicht durch eine solche Wirlung des Mikrotommessers hervorgerufen ist, dass sie eben thatsächlich nicht grösser sind, wie man auch auf den längs- 
getroffenen elastischen und leimgebenden Faserbündeln sehen kann.

2) Wenn die Kerne dureh Wanderung an die Wunde gelangten, so müssten wir sie innerhalb der präformirten Spalten oder Saftlücken antreffen, sie liegen aber wie oben ausgeführt auch dort, wo sie reibenweise angeordnet sind, immer neben dem Spalt in der diesen begrenzenden Grundsubstanz oder gar ritten in den Gewebsbündeln.

3) Gelangten die Kerne wirklich durch Auswanderung aus den Gefässen in das Gewebe, so müsste man bei einer so massenhaften Emigration Kerne in allen Stadien des Durchtretens finden. Trotzdem wir auch hieraufhin viele hunderte von Präparaten durchmusterten, trafen wir nur einmal ein leukocytenähnliches Gebilde, das man seiner Lage nach als auf der Auswanderung befindlich gelten lassen konnte, nicht musste.

4) Gegen eine Wanderung der Gewebszellen spricht ausser den Punkten 1 und 2 der Umstand, dass nirgends in der Nachbarschaft der Wunde eine Zellverminderung zu bemerken ist, die bestehen müsste, wenn eine irgendwie erhebliche Wanderung der präexistirenden Zellen stattfände. So bliebe denn als ultimum refugium eine Theilung und Wucherung der Gewebszellen. Abgesehen davon, dass für einen sehr grossen Theil der kleinsten Chromatinformen, besonders soweit sie isolirt inmitten eines Gewebsbündels in der Mittelebene nicht ganz dünner Schnitte liegen, diese Erklärung von vornherein hinfällig wird, muss auch für die überwiegende Mehrzahl der andern die Entstehung durch Zelltheilung als nicht zutreffend zurüekgewiesen werden, weil die Kerne vielfach durch grosse Abschnitte unveränderter Grundsubstanz von einander getrennt sind. Ausserdem findet man erst vom dritten Tage ab eine irgendwie ins Gewicht fallende Anzahl von Mitosen im Bindegewebe.

Bilder also, wie wir sie in Figur 1, 2, 3, 4 u. a. abgebildet haben, können weder durch die Emigrationstheorie noch durch die Zelltheilung, noch schlechtweg durch Wanderung von Gewebszellen erklärt werden. Diese Bilder deuten vielmehr darauf hin, dass noch auf andere Weise Zellen im Gewebe entstehen können. Einen neuen Beweis hierfür geben unsere Bilder. Man hat allerdings dieser Erklärung vorgeworfen, dass sie der Specu- 
lation and Willkur Thür und Thor offne, indern sie die alte längst verworfene Lehre vou der generatio aequivoca zar Vorausetzung hätte. Dem ist aber nicht so, eine generatio aequivoca d. h. Entatehung lebender Materie aus dem todten Stoffe, liegt hier gar nicht ror. Die Grundsubstanz ist nioht todt, sie lobt and nimmt Theil am Stoffwechsel and kann sich, wie dies z. B. bei der Altersinvolution der Knorpels anerkannt ist, völlig verändem.

Wenn wir, wie obon erwähnt ist, als bewiesen erachten, Aass die Grundsubstanz entsteht nicht durch Abscheidung eines todten Materials von Seiten der Zellen, sondern durch eine Umwandlung der Zellen selbst, dass somit die Grundsubstanz die Form ist, in der die Mehrzabl der Zellen im menschlichen Organismus unter gewöhulichen Verhältuissen existirt, so geht Grawitz nur einen Schritt weiter and zieht gewissermassen die Consequenz aus diesem Satze, wenn er behauptet, dass der Zellencomplex, der die Grundsubstanz darstellt, bei Ernährungsstörungen wieder zur Lebensform wirklicher Zellen zurückbehren kann.

Veberall da also, wo ein Reiz statt hat, wo durch einen gesteigerten Saftstrom in erhöhtem Maasse Nährstofe den Geweben zugeführt werden, sehen wir, 1) dass, wie es Virchow in der Cellularpathologie lehrt, die Zellen sich activ betheiligen, lass die unvollkommenen, kleinen, im Ruhezustand äusserst protoplasmaarmen Bindegewebskörperehen sich vergrössem (nutritive Reizung), 2) dass die Intercelluiarsubstanz wahrscheinlich unter Aufnahme von Ernährungsmaterial und Umlagerung ihrer kleinsten Theile in einen zelligen Zustand übergeht, der allerdings eine gesteigerte Ernährung erfordert, dafür aber einen wirksameren Widerstand und erhöhte Leistungen gestattet.

Wir finden also, dass in den Theilen, wo die Reaction zuerst eintrat, und die Veränderungen demgemäss am weitesten vorgeschritten sind, von der Grundsubstanz nur noch ein Gerüstwerk erhalten ist, das in seinen Maschen Zellen bezüglich Kerne enthält. (Fig. 8 u. 9).

Aber nicht diejenigen Abschitte sind für den Vorgang der Zellenbildung aus der Grundsubstanz charakteristisch, in welchen zwischen den einzelnen Elementen nur noch ein feines Roticulum 
von mucinöser oder albuminöser Substanz übrig geblieben ist denu diese feinsten Fäserchen können auch auf eine Gerinnung einer vorher flüssigen Zwischensubstauz bezogen werden - sondern solche Stadien, in welchen sowohl die kleinsten Kernformationen, als die grösseren Endothelkerne und endlich die Kerne mit spindeligem, rundlichem, sternförmigem Zellenleib ringsum von unveränderter homogener oder fibrillärer Grundsubstanz umgeben sind.

Nach dem Beobachteten geht die Zellenbildung in der Weise vor sich, dass sich neben den Saftspalten zunächst ein kleinstes Chromatinhäufchen, nicht grösser als ein Coccus oder Bacillus oft im Verlaufe von Zollfortsätzen oder Fasern färbt.

Diese sich intensiv färbenden Theilchen nehmen an Grösse zu, es treten auch wohl, wenn der Prozess stürmisch verläuft, gewöhnlich dort, wo mehrere Saftspalten zusammenstossen, mehrere unmittelbar neben einander auf, und, indem sich dann aus der Grundsubstanz ein hellerer Hof um sie bildet, entstehen die lenkocytären Formen (Fig. 9L), die aber in vielen Fällen keine wirklichen Zellen, sondern nur Kerve mit zusammengeklumpter Chromatinsubstanz und deutlich färbbarer Kernmembran darstellen.

Doch müssen wir bemerken, dass die angetroffenen Bilder ausserordentlich mannichfaltig sind, und vorstehendes nur einen Typus der Zellenbildung treffen kann. Vielfach sehen wir dort, wo die Reaction ruhiger erfolgt, Chromatinformationen, die von ihren kleinsten Anfängen an dem Typus der Endothelkerne folgen, gleichsam Endothelkerne en miniature sind (Fig. $9 \mathrm{k} \mathrm{u}$. e).

Dass wirklich die kleinen Formen zu Zellen werden, beweist Ort und Zeit, in der man sie trifft. So finden wir in den ersten Stunden nach der Verletzung vorwiegend und in grosser Mehrzahl die ganz kleinen Formen; in den Präparaten von längerer Heilungsdauer ist immer die Grenze der nkleinzelligen Infiltration", die Vebergangszone vom pathologischen zum normalen der Ort, wo man sie hauptsächlich trifft. Sie sind also weder im normalen noch in dem schon ganz zellig geschmolzenen Gewebe in grösserer Zahl vorhanden, sondern immer nur da, wo das Gewebe im Beginne der Reaction, der zelligen Umwandlung steht. 


\section{Tage.}

Zur Untersucbung wurden wieder die Wunden ron zwei Herren verwandt. Die beobachteten Vorgänge deckten sich in beiden Fällen fast vollkomaen, nur in einzelnen winderwichtigen Punkten wichen sie ans oinander. So War z. B. in dem einen Falle die Wunde in ihrea ganzen Verlanfe dureh vine Epithelbücke geschlossen, wähend hingegen dies bei der anderen mur erst an den Enden der Fall war. In der Mitte dagegen, wo die Wunde wuch nach der Tabt noch ein wonig klafte, war zwar schon von dem Epithel beider Wundränder je ein Zapfen nergebildeter Epideruiszellen über den Wundrand vorgeschoben, eine Vareinigung war jedoch noch nicht erfolgt. In allen diesen Fällen war der Epithelsaum des einen Wundrandes in die Wunde eingebogen.

Unterhab dieses Epithels ist kein Spalt mehr vorhanden, aur einzolne gelbbraune Blutcoagula zoigen, dass hier früher die Wundränder njeht unnittelbar an einander lagen. Im Uebrigen ist der Verlauf der Wunde gecennzeichnet durch ein Netzwerk, wie es Fig. 8 veranschaulicht. Dicke, durch Saffranin intensiv roth gefärbte Fasern ziehen den Wundrändern parallel in die Tiefe, sie sind verbunden durch feinere, quer verlaufende, ebenso gefärbte Wasern, vou denen sich ein Maschenwerk feinster anastomosirender rother Fädchen und Linien abzweigt und so de grossen Felder in kleine Abschnitte theilt. In jedew dieser kleinen Färher liegt je eine Zelle oder Kern I).

Bei intensiver Saffraninfärbung sieht man, wie sich die Fäden des Maschenwerkes fortsetzen in die roth gefärbten T'rennungslinien der Bindegewebsbündel, wo sie dann allmählich in ungefärbten Linien enden, wie dies Fig. 8 darstellt. In Verlaufe der gefärbten Theile findet man hin und wieder Kernanschwellungen, ähnlich wie dies Fig. 3 und 4 zeigen.

Die Zellen, welche in den Fächorn liegen, stehen zum Theil mit ibrem Protoplasma noch deutlich in Verbindung mit den Fasern, manchmal sieht man auch Bindegewebe und Kerne in einem und demselben Fache. Die Kerne zeigen in der äberwiegenden Mehrzahl endothelialen Cbarakter, daneben findet man allerdings eine Anzahl mehrkerniger Zellen, die eine gewisse Aehnlichkeit mit. Leukocyten haben, rielfach aber durch Grösse und Beschaffenheit ihres Zellleibes davon unterschieden sind.

Un den eigentlichen Wundspalt namentlich im Bereich der Cutis ist die Zahl der Zellen gering, dagegen im Fettgewebe und in der ganzen Umgebung der Nähte ist eine breite Zone von so reichlicher Kernanhâufung, alas das Bild vollkommen der beginnenden eitrigen Schmelzung gleicht

1) Der schon oben erwähnte Atlas der pathologischen Gewebelehre von Grawitz enthält in dem Kapitel äber die Wundhelung 2 Photogrambe gerade dieser Präparate, die in noch sebönerer Weise als unsere Skizzen das verklebende Netzwerk, die Gestalt und das Verhältniss der Zellen, Kerne und Bälkchen zu einander, den Zusammenang mit dem weniger veränderten Gewebe der Nachbarschaft veranschaulichen. 
(vgl. Fig. 9). Die Zellen liegen aber nicht etwa regellos zerstreut, sondern lassen im derben Gewebe deutlich erkennen, dass sie am reichlichsten in den loseren Zügen um die quer und schräg getroffenen derben Faserlager angeordnet sind und zwar so, dass diese im Bogen verlanfenden Züge nach dem Wundspalt zu immer reichlichere Kerne enthalten, wäbrend sie mit der Entfernung immer schmäler werden und in ibrer Nähe eine Zerklüftung und Zellenanhäufung längs der Spalten der derberen Bündel erkennen lassen, so dass sich von der Wunde aus der breite Strom der Kerne allmählich im Verlaufe der Spalten zwischen den Bündeln der Cutis verliert. Nach Mitosen sucbt man in diesen Zellen vergebens. Ueberfaupt findet man im Bindegewebe zwei Tage nach der Verletzung böcbstens ganz vereinzelt einmal in den Endothelien der Blutgefässe eine Kerntheilungsfigur, wäbrend hingegen die Epithelien des Rete, der Haarbälge und der Drüsen sehr viel öfter karjokinetische Figuren enthalten. Leukocyten in stadio emigrationis haben wir in den Blatgefässwandungen an keiner Stelle trotz eifrigen Suchens angetroffen.

\section{Tage.}

Senkrechte Durebschnitte zeigen, dass die Wunde überall durch Epithel verschlossen wird, während bingegen eine vollkommene Vereinigung des Cutisgewebes noch nicht erfolgt ist. Ein feines, roth gefärbtes Netzwerk, das das Bindegewebe beider Wundränder verbindet, schliesst ein ziemlich grosses Blutgerinnsel ein, in welch letzterem also ein ähnliches Maschenwerk nicht vorhanden ist. In dem Fettgewebe verbreitert sich dieses intensiv roth gefärbte Netzwerk trichterartig.

In den Maschen liegen nur ausnahmsweise Zellen mit mehreren intensiv gefärbten Kernen, die meisten derselben entbalten einen bläschenförmigen Kern mit färbbarer Kernmembran und zierlichem Chromatingerüst und einem oder mehreren Kernkörperchen. Auch Riesenzellen mit mehreren endothelialen Kernen gehören nicht zu den Seltenheiten. Auch die Wundränder enthalten yorzugsweise Kerne vom Endotheltypus in der im vorigen Protocoll. angegebenen Anordnung.

In der Wandung der Blutgefässe sind zahlreiche Mitosen enthalten, oft sieht man deren drei in einem Gesichtsfeld. In den eigentlichen Bindegewebskörperchen, sowie in den Bezirken der zelligen Schmelzung und in den Zellen des Netzwerkes sahen wir keine Karyokinesen. Wohl aber enthielten die verschiedenen Epitbelien eine ganze Reibe sebön fixirter Mitosen. An den Gremzen der zellig geschmolzenen Gewebspartien trifft man vielfach kleinste bläschenförmige Kerne im Verlaufe der leimgebenden Faserbündel und der elastischen Fasern, wie dies Fig. $9 \mathrm{k}$ und e zeigt.

Der Seidenfaden der Naht wird an seiner Aussenseite von einem mehrschichtigen Zug von Epithelien begleitet, der dem Verlauf des Stichkanals folgend, sich ziemlich weit in die Tiefe senkt.

Epikrise. Aus dem Angeführten ist ersichtlich, dass die Präparate vom zweiten und dritten Tage sich von den Schnitten 
aus den früheren Stadien der Weilung aicht sowohl durch die Zahl der Kerne an der Wundspalte als vjelmehr durch das A ussehen derselben unterscheiden. In den frühten Stadien des Prozesses äberwogen die ganz kleinen Formen, später fand man hauptsächich die polynucleären lenkocytenähnlichen Gebilde, am zweiten und noch mebr and dritten Tage sind diese aus den Präparaten mehr und mehr verschwunden und an ihrer Stelle liegen jetzt protoplasmareiche Zellen mit endothelialem Keru.

Fischer') hat sich dies so zu erklären versucht, dass or an ersten Tago nur mehrkernige, später anch einkernige Leukocyten und endich danach auch Gewebszellen bezüglich AbkömmJinge dersalben in die Wunde einwandern lässt. Nach seiner Ausicht sind diese in den verschiedenen Tagen verschieden aussebenden Elemente in der Wande auch wirklich verschiedene Zellindividuen.

Wir könnev uns dieser Anschaunng, für die sich Beweise kaum erbringen lassen dürften, nicht anschliessen. Der unmittelbare Zusammenhang vielmehr, in dem sowohl in den ersten Stunden die ganz kleinen Formen, sowie später die leulocytären Blemente, wie endlich auch in den weiteren Stadien der Heilung: die endothelialen Zellen mit der Nachbarschaft, der Grundsubstanz, den elastischen Fasern und den Balken des fibrinoiden Maschenwerks stehen, führt mit zwingender Nothwendigkeit zu dem Schlusse, dass diese verschiedenen Formen nur verschiedene Sntwickelungsstadien ein- und derselben Zellindividuen sind. Die Beziehung, in der alle diese mit ihrer Nachbarschaft stehen, lässt als einaig natürlich and wahrscheinlich nur die Annahme zu, dass die höher entwickelten Kerne aus den niederen Formen hervorgehen, dass durch eine Umlagerung der Chromatinsubstanz aus den leukocytenähnlichen die epitheloiden Kerne entstehen.

Auch Cohnheim konnte sich dieser natürlichen and berechtigten Schlussfolgorung aus den Bildern wicht ontziehen, er schloss deshalb und lehrte in früheren Jahren, dass die Leukogyten sich weiter entwickeln und Gewebe bilden könnten. Erst nachdem dann auf anderem Wege gezeigt war, dass die Gewebsbildung in der von Virchow festgestellten Weise durch nfixe Gewebszellen " erfolgt, musste man, da man ja die polynucleären

1) S. 0 . 
Elemente fälschlicherweise als ausgewanderte Leukocyten voraussetzte, nach so geschraubten Erklärungen suchen, dass die verschiedenen Zellen sich gewissermaassen militärisch bei dem Heilungsgeschäfte ablösen. Cohnheim hat richtig beobachtet und gefolgert, dass die verschiedenen Formen auseinander hervorgehen, falsch war nur die Voraussetzung, dass dies Leukocyten seien. Als nun nachher'der Endschluss "Leukocyten bilden die Gewebe" als unrichtig zurückgewiesen wurde, so liess man leider vicht die irrige Prämisse fallen, dass alle kleinen Rundzellen farblose Blutkörper seien, sondern suchte auf ihr fassend nach neuen unrichtigen und unnatürlichen Erklärungen für die mannichfachen Bilder.

Dass Umlagerungen und Umgestaltungen des Chromatins vorkommen und, rein theoretisch betrachtet, denkbar und möglich sind, das zeigen zur Genüge die Vorgänge bei der mitotischen Kerntheilung. Auch hierbei ist das hauptsächlich in die Augen fallende ein Wechsel in der Anordnung der Chromatinsubstanz.

Weniger als in diesem Punkte konnten wir über die Vorgänge bei der Epithelisirung zu der gewünschten Klarheit gelangen.

\section{Tage.}

Die Wunde ist vollstïndig durch eine viele Lagen dicke Schicht von Epithelien geschlossen; über. der neugebildeten, bogenförmig in die Wunde sich senkenden Epithelbrücke ist eine verbornte Schicht noch nicht vorhanden. In der Wunde befindet sich reichliches Blutextravasat, das sich in die Spalten und Lücken der Nachbarschaft fortsetzt, und besonders die loseren Gewebslagen um die Fettläppchen herum aus einander gedrängt bat. Begrenzt wird das Gerinnsel an der einen Seite von einem intensiv roth gefärbten Netz, das aber nur bis in die äussersten Randpartien des Gerinnsels eindringt. Der grössere Theil ist absolut frei von einem äbulichen Maschenwerk. Dieses ist in den vorliegenden Präparaten sehr breit und zeigt eine noch feinere Struktur wie in den Präparaten anderer Tage. Das Gewebe der Nachbarschaft zeigt im Gegensatz zu Wunden aus früberen Tagen auffallend wenige Veränderungen.

In dem Gitterwerk liegen überwiegend Zellen mit endothelialen Kernen und körnigem, zartem, verschieden grossem Protoplasmaleib. Dieser umgiebt entweder den Kern wie ein schmaler Saum oder sendet in die verschiedensten Richtungen mannichfaltig geformte Fortsätze, die entweder mit einander communiciren, oder in anderen Fällen mit den rothen Balken des Maschenwerkes in Verbindung stehen, oder ganz almmählich in die unveränderte Grundsubstanz übergehen (Fig. 9). In den Fibrinfasern sind kleine Kerne 
eingeschlossen, wie in Fig. 8 E. Nicht gerade selten trift man Riesenzellen wit drei, vier oder noch mehr endothelialen Keruen. Typen aller dieser Zellormen sind in Ziegler's Lehrbuch der allgemeinen Pathologischen


gebung der Wunde sioht man Zellenanhäufungen un Drüsen und Blutgefïsse und in besonders reicher Zahl um den Seidenfaden herum. Sonst liegen vereinzelt neben den Spalten häufig reihenweis geordnete Kerme meistens rom Wndotheltypus. Viele derselben sind ron einem Hof iömigen Protoplasmas umgeben wie dies Fig. $9 \mathrm{p}$ sehr schö zeigt, andere liegen noch in anveränderter Grundsubstanz, wieder andere sind go blein, dass man sio eigentlich kanm als Kerne bezeichnen kann. Die elastischen Kasern enthalten anch vielfach endotheliale Kerne and Zellen, wie dies Fig. 6 domonsirint.

Das Bluteoagulum im Wundspalte ist wenig verändert; wan sieht nur einzelne leukocytåre Gebilde darin. Mitosen sind in den Zellen zwischen dem Gitterwerk nur vereinzelt, dagegen sieht man solehe in ziemicher Menge in den Wandungen kjeiner Blutgefässe und Capiliaren, hin und wieder auch in den Zellen des Bindegewebes. Auch in Epithel liegen einige gut fixirte Mitosen. Die Zellen der die Wunde deckenden Epidermissehicht sind theils gross and prall, theils mebr platt und spindelformig.

\section{$6 \mathrm{Tage}$.}

Yie fruhere Wunde ist durch eine breite Epithelbräcke vollständig geschlossen, deren oberer Rand in dem iveau der verhornten Epithelien der Nachbarschatt liegt. Nach unten setzt sich ein trichterformiger Epithelzapfen in den früheren Wundspalt hinein fort. In der Wunde ist das Fibrinuetz bedeutend geringer wie an den vorhergehenden.Tagen. Auch in diesen Präfaraten findet man einen grossen Bluterguss 3 wischen den Wundrändern und in den Safispalten der unteren Gewebslagen. In der Nachbarschaft der Wunde triff man besonders um Blutgefässe und Drüsen eine Vermehung der Rerne.

In dem Fibrinnetz sind runde und sternfömige, grosse, protoplasmareiche Zelien mit Endothelkernen, in denen zahlreiche Mitosen vorbanden sind. Die Zellen stehen durch ihre Ausläufer vielfach in Verbindung. Man findet auch Riesenzellen in allen Grössen, grosse Protoplasmahaufen unit zwei bis vielen bläschenförmigen Kenen. Viele von diesen Zellen enthalten in ibrem Leibe kleine, durch Osmíumsätre scbwarz gefärbte liettiügelchen oder Kern- and Fibrintrümmer. Vereinzelt trifit man anch noch Gebilde, die den Leukocyten nicht unähnlich sind. In dem Blutgerinnsel sieht man nur wenige lenkocytäre Elemente, so dass von einer nennenswerthen Einwanderung ron weissen Blutkörperchen in das Extravasat nicht die Rede sein kann.

In der Nachbarschaft der Wurde siebt man auch za dieser Zeit an und in den elastischen Fasern grosse Endothelkerne mit regeluässig angeordnetetn Chromatingerüst (Fig. 6). Einzelne Doppelsterne liegen so, aass sich die 
Zellsubstanz noch in den graublawen Farlen einer elastischen Faser hinein verfolgen lässt. Auch in dem weicheren Bindegewebe um die Talgdrüsen oder Nerven oder Blntgefässe, wo in den ersten Tagen nur ganz kleine, stark gefärbte, längliche, dreieckige oder kleine ovale oder aus mebreren Fragmenten bestehende Kerne beobachtet wurden, finden sich in mehreren Reihen angeordnete, anastomosirende Sternzellen mit grossen Endothelkernen, die ebenfalls Mitosen enthalten. Zahlreiche prachtroll fixirte Mitosen trifft man auch in den Epithelien, sorohl in denen des oben bescbriebenen, in die Wunde bineinragenden Zapfens, wie auch in denen der Nachbarschaft. $\mathrm{Zu}$ bemerken ist auch, dass diese Mitosen keineswegs nur in den alleruntersten Lagen des Epithelsaums, sondern hin und wieder auch in Zellen aus der Mitte des Rete Malpighii gefunden werden.

Epikrise. Wir sehen in diesen Präparaten die Weiterentwickelung der aus der Grundeubstanz entstandenen Kerne und Zellen. Die anfangs überwiegend angetroffenen Kerne mit dem zerklumpten Chromatin entwickeln sich durch Umlagerung ihrer Chromatinsubstanz und bilden nun gleichsam ein Attractionscentrum für die Bildung von Protoplasma. Indem die Kerne körniges Protoplasma um sich herum sichtbar werden lassen, werden sie nun wirkliche Zellen, die mit den Nachbarzellen in Verbindung stehen oder gar damit verschmelzen, und so gelegentlich Zellen mit mehreren Endothelkernen, also Riesenzellen, bilden. Diese Art der Entstehung von Riesenzellen ist nach dem Beobachteten sehr wahrscheinlich, ohne dass wir damit irgendwie andere Möglichkeiten der Bildung von Riesenzellen ausschliessen wollen.

\section{Tage.}

In den senkrecht zur Hautoberfäche und der Längsrichtung der Wunde angefertigten mikroskopischen Schnitten ist die Stelle der Wunde gekennzeichnet durch eiren dreieckigen in die Tiefe gebenden Epithelzapfen, an den sich nach unten eine sehr zellenreiche Zone mit spärlicher Zwischensubstanz und stellenweise erhaltenen Resten des früheren fibrinoiden Masebenwerks anschliesst. Diese Zellenpartien durchsetzen auch auf eine ziemlich grosse Strecke das Gewebe der Nachbarschaft, indem von dem früheren Wundspalt ab nach beiden Seiten hin anfangs breite, weiterhin schmaler werdende Zellreihen sich zwischen die derberen Bündel der relativ wenig veränderten Grundsubstanz fortsetzen. Sonst fällt besonderer Zellenreichthum in den seitlichen Partien des Sebnittes noch auf in der Ungebung der Drüsen, Fettläppchen, Nerven und Blutgefässe. Das Fettgewebe zeigt in der näheren Umgebung des früheren Wundspaltes anch bedeutende Zellenvermehrung.

Archiv f. pathol. Anat. Bd. 134. Hft. 3. 
Bei starker Vergrösserung seben wir in der zellenreichsten Portie viele sich freuzende Züge yon anastomosirenden spindel- und sternförmigen Zellen rait bläschenförmigen Kernen. In diesen ist das zierliche Curomatingeräst wit den Kerntörperchen brillant erhalten, mehrere eathalten schön fixirte Uitosen. Einzelne ron den Zellen zeigen in ibren letzten Enden schon fibrilläre Structur.

Anch findet man ganze Reiben schlanker Spindelzellen in unmitteibarem Zusarmenhang, so dass man eine eigentiche Grenze zwischen dex einzelnen Zellen nicht mehr erkenuen kann. Zuweilen gelingt es, eine derartige Zallreihe bis zu einem Faserbündel der Grundsubstanz zu verfolgen, dessen disecte Fortsetzung die Zellen zu sein scheinen.

Derartige, direct zusammenbingende Zellen, zuwellen in mehreren concentrischen Reihen, finden wir auch vielfach uw die Talgdrüsen, Nerven und Gefässe herum.

Doch sei nicht unerwähnt, dass wir hart an dem zelligen Bezirk noch wieder gauz ähnliche Bilder finden, als wie wir sie nach $3 \frac{3}{4}$ Stunden za seben bekommen und dort ausfübrlich beschrieben haben. Es berechtigt dies noeb zn dem Sehluss, dass die Reaction in Gewebe noch fortschreitet und noch weitere Kreise desselben mit in den Heilungsprozess einbeziebt.

\section{Tage.}

Wunce der Haut der medialea Seite des linken Oberarmes. Die Wunde wurde durch Seidennähte geschlossen. Am 6. Tage wurden die Näbte entfernt.

Bei scbwacher Vergrösserung seben wir am oberen Rande der Schnitte uberall einen breiten Epithelsaum. Ungefähr in der Nitte desselben finden. wir eine weniger dicke Schicht verhornter Epithelien. Entspreeheud dieser Stelle ragt ein Zapfen des Epithels tiefer in das Bindegewebe binein. In wenigen Schnitten, die offenbar durch den Stichkanal der Naht gehen, sioht san seitlich yon diesem breiten Epitbelzapfen einen langen, schwalen Streifen yon Epithelien bogenförmig von dem Rete in die Tiefe und gegen. die Mitte des Schnittes binziehen; dieser Streifen entspricht dem früheren Stichtanal der Naht.

Die Seitenpartien des Schnittes scheinen aus nicht sehr verändertem cutisgewebe zu bestehen. Sie werden ron einander getrennt duch einen breiten Streifen total anders aussehenden Gewebes, das avs zabllosen Zellen mit geringer $Z$ wischensubstanz und in den unteren Partien aus dunkelroth gefärbten Resten des früheren Eibrinnetzes zusammengosetzt wird. Es fehlen ganz in diesem Streifen die grossen Felder der derben Bindegewebsbündeí, sowie die elastischen Fasern der Seitenpartie.

In diesem mittleren Streien, der der ursprünglichen Wunde entspricht, fuden wir bei stärkster Vergrösserung prachtvoll ausgebildete, grosse Spindelzellen oder Sternzelien mit ovalem oder seblankem, endothelialem Kern, jer ein schön erhaltenes Chromatinnetz und ein oder webrere Kernkörperchen enthält. Vielfach sehen wir die Spindelzelten, obne dass ibre Grenzen erkennbar sind, zu einer langen Reihe znsammengelagert, so dass man den 
Eindruck empfängt, als habe man einen langausgezogenen Streifen körnigen Protoplasmas vor sich, in dem in bestimmten Absätzen ein ovaler oder schlanker, bläschenförmiger Kern liegt. Theilweise treffen wir auch Zellen, deren Protoplasma sich schon differenzirt und besonders an seinem Ende fibrilläre Struetur zeigt oder unmerislich nod ganz allmählich in die neugebildete Grundsubstanz übergeht, Fährend der Kern noch in seiner Gestalt erhalten ist. Vou anderen Zellen ist als Kern nur noch eine recht lange, blasse Spindel geblieben, die in einem Faserbündel liegt.

Kommt man zur Grenze zwischen dem neuen und alten Gewebe, so tritt auch bervor, dass die neugebildete Grundsubstanz sich noch wesentlich von der alten unterscheidet. Diese sieht viel gleichnässiger und glänzender aus wie jene. Sofort fallen in dem alten Gewebe auch die glänzenden, elastischen Fasern in die Augen, die sich nach dem Wundspalt zu theilweise direct in eine Zellreihe auflösen. Weiter entfernt trifft man anch elastische Fasern, die in ihrem Verlauf eine schön ausgebildete Endothelzelle tragen. Einmal fanden wir sogar eine Mitose in einer solehen spindelförmigen Zelle, deren Enden direct in eine gequollene elastische Faser übergeben und die wir in Fig. 6 abgebildet haben. In der Wunde selbst trifft man auch vereinzeite Mitosen, besonders, wenn man sich den Stellen nähert, in denen noch Reste des Fibrinnetzes gefunden werden. In der Nähe dieses Netzes treffen wir auffalligerweise noch Bilder, wie wir sie sehon aw ersten Tage gesehen und beschrieben haben. Nan geht wohl nicht fehl, anzunehmen, dass hier noch breitere Gerrebsschichten für die Heilung in Anspruch genommen werden.

An anderen Stellen, wo die Einschmelzung des Gewebes sicherlich nicht fortschreitet, seben wir dennoch anch häufig solche halbfertigen Zellen oder Kerne in der relativ unveränderten Grundsubstanz. Hier bandelt es sich denn wobl um unvollkommene Rückbildung der Grundsubstanz in Zellen. Zuverlässig müssen und werden wir Fasern treffen, bei denen es nicht zur Ausbildung der vollständigen Zelle gekommen ist. Eben so gut wie wir im normalen Bindegewebe Kernfasern finden, bei denen die Umwandiung der Zelle in Grundsubstanz auf einer gewissen Uebergangsstufe stehen geblieben ist, treffen wir im gewucherten Bindegewebe kernbaltige Fasern, die, wenn die Entzünd̊ung zum Stehen kommt, sich noch nicht völlig in Zellen verwandelt baben und den rein zelligen Zustand auch nie erreichen, sondern vorher wieder zur Rube gehen.

An einigen Stellen finden sich auch in dieser Periode neben dem Fibrinnetzwerk zerfallene Kerne und Zellen, Fett- und Pigmentkörnchen, welche in grösseren protoplasmareichen Zellẹ eingeschlossen lagern. Dass aber nicht alle Trümmer nur durch Zellen weggeführt werden, gebt daraus hervor, dass man, abgeseben von der jungen Narbe, in dem Nachbargewebe solche Pigmenthäufchen antrifft, welche in schlanken Gewebszellen liegen, die ibrerseits an Saftspalten angrenzen, aber nur zum Theil Zellenprotoplasma besitzen, zum grösseren Theil der ruhenden Grundsubstanz angehören. 


\section{Tage.}

Wuade aus der Jateralen Seite des linken Oberardes. De Wunce wurde mit Seide genäht. Die Năhte wurden am 6. Tage entfern.

Wie bei den Präparaten rom 10. Tage, so unterscheidet sich auch hior de Narbe in der Mitte des Sehnttes duch Fäbong und Strubtur von dem relativ unveränderten Cutisgewebe der Seitenpartien. Es fehlen in der Narbe die grossen Feider derber Bindegewebsbündel, sowie die elastischen Fasern, aberall bestehen nur kleinere Felder von Grundsubstanz, denen Zellen anliogen oder Kerne eingelagert sind. Ueber die junge Narbe zieht Ner Lpithelsaum glatt binweg. Papillen sind nicht ausgebildei. Bei starker Vergrösserung sieht man in der mittleren Partie vorzugsweise langgestreckte Spindelzellen, die entweder unter einander durch Aus?äufer in Verbindung steben oder aber an dem einen Ende in einen Faserang ubergeben.

Vielfach sehen wir langgestreckte Spicidelzellen mit schwach färbbarem, bläschenfömigem Kern mit ihrer Längsseite an Faserbindeln liegen. Das Protoplasma derselben geht ganz unmerkich in die Grandsubsianz über. Die neugebildete Grundsubstanz der Narbe erscheint viel beller und weicher wie die derben Bindegewebsbündel in dem Cutisgewobe. Noch hänfiger wie am 10. Tage treffen wir etwas grössere Felder nongebildeter intercellularsubstanz und lango, ganz schmale Spaiten, die Kermieste erkennen lassen. Vielfach zeigen die leicht wellig verlaufeaden Faserbündel eine fibrilate Streifung.

An der Grenze zwischen altem und nenem Gewebo gelingt ss leicht, Zellreihen zu finden, die sich direct in eine dunkelgefäbte, elastische Faser les Cutisgewebes fortsetzen.

In dem neugebildeten Gewebe und der Nacbbarschaft findet man vielfacb stark erweiterte, prall gefällte Blutgefässe. Dinmal wurda in dem neuen Gewebe eine Mitose beobachtet. Riesenzellen kamen ofters vor.

Epikrise. Aus der Besehreibung der Präparate vom achten, zehnten und zwanzigsten Tage erkent man, dass nummehr die aigentliche Narbenbildung vor sich geht. Wir sohen aber auch daraus, dass dieser Uebergang des Granulationsgewebes zu Narbengewebe, das heisst; die Umwandlung der Zellen zar Grundsubstanz ganz andere Bilder giebt, als der umgekehrte Vorgang, d. h. der Uebergang eines fertigen Gewebes in den zelligen Zustand. Hierbei sehen wir im Anfang den Grenzen der grossen, später auch der kleineren Bündel kleinste intensiv färbbare Chromatingebilde anliegen, während andere Bündel, in denen die zellige Umwandlung schon weiter fortgeschritten ist, grössere Kerne oder vereinzelte Zellen, meist vom Typus der Leukocyten, enthalten. Man trifft also grosse Felder unveränderter Grandsubstanz, solche mit kleinsten Kernanfängen und endlich andere schon völlig zellig umgewandelte dicht neben einander. 
Bei der Narbenbildung hingegen sieht man in ziemlich regelmässigen Abständen hochentwickelte Kerne von Spindelform mit oder ohne Zellleib kleinen Feldern von zarter Grundsubstanz anliegen. Nirgends findet man, dass an einer Stelle etwa die Bildung kernloser sklerotischer Bindegewebsbündel vollendet ist, während noch unmittelbar daneben Granulationsgewebe ohne jede fibrilläre Zwischensubstanz vorhanden ist.

Was nun die Bildung der Grundsubstanz anbetrifft, so schliessen wir uns im Ganzen der folgenden, durch vorzügliche Abbildungen illustrirten, treffenden Schilderung von $Z i$ egler ${ }^{1}$ ) an: „Hat ihre (der Fibroblasten) Zahl eine gewisse Höhe erreicht, so beginnt die Bindegewebsentwickelung, d. h. die Bildung der fibrillären $Z$ wischensubstanz. Dieselbe entwickelt sich theils unmittelbar aus dem Protoplasma der Bildungszellen, theils aus einer bomogenen Grundsubstanz, welche sich zuvor aus den Fibroblasten gebildet hat. Im ersteren Falle treten sowohl an den Längsseiten als den Enden der Bildungszellen feine Fasern auf, welche sich mit Fasern aus benachbarten Faserzügen vereinigen. Die Richtung und Grösse der dadurch entstehenden Faserzüge ist unabhängig von der ursprünglichen Configuration und Lagerung der Bildungszellen; meist ist die Richtung der Faserzüge über grössere Strecken dieselbe. Bei einer gewissen Mächtigkeit der Fibrillen macht die Faserbildung Halt, die Reste der Bildungszellen mit ihren Kernen bleiben als fixe Bindegewebszellen bestehen und lagern sich der Oberfläche der Fibrillenbündel an."

Bis auf den letzten Satz stimmt diese Schilderung mit dem Resultate meiner Untersuchungen überein. Ich muss nach dem von mir Beobachteten diesen Satz folgendermaassen modificiren: Von den Bildungszellen bleibt ein Theil ihrer Kerne mit oder ohne Protoplasmaleib als sogenannte Bindegewebskörperchen bestehen, während die grosse Mehrzahl derselben völlig auch mit ihren Kernen in den Faserzustand übergeht.

Hätte $Z$ iegler recht, so müsste jede Narbe mit der Zeit hypertrophisch werden. Denn man sieht bei der Untersuchung

3) Ziegler, Lehrbuch der allgemeinen pathol. Anatomie und Patbogenese.

VI. Aufl. 1889. S. 213. 
Goch späterer Stadien, dass die Narbe Immer Bernärwor wird, d. h. dass die Bindegewebskörperchen dureh immer grössere Gelder von Intercellularsubstanz getrennt werden. Es wird also, wenn wirklich alle die Fibroblasten in der Narbe als Bindegewebszellen erbalten bleiben, welche ursprünglich fast unmittelbar aneinanderliegend, nachher durch Zwischensubstanz getrennt, theilnahmen an der Narbenbildung, die Entfernung aller Fibroblasten von einander immer grösser, und damit muss der Raum, den die Gesammtheit derselben einnimmt, in demselben Masse wachsen.

Es müsste also makroskopisch die Narbe mit der Zeit an Volumen gewinnen. Dem widersprechen aber die thatsächlichen Vorgänge an den Narben. Es ist allbekannt, dass die Narben schrumpfen d. h. mit der Zeit an Volumen abnehmen. Blieben aber nun alle Fibroblasten oder alle Bindegewebszellen einer jungen Narbe weiterhin bestehen, so müssten sie mit fortschreitender Schrumpfung näher an einander rücken, statt dass, wie dies thatsächlich der Fall ist, der Abstand der einzelnen Bindegewebskörperchen von einander grösser wird und zwar derart, dass man in sehr alten Narben oft grosse Felder ohne jeden Kern antrifft.

Diese einfache Betrachtung zeigt, dass die absolute Zahl der Bindegewebszellen in einer jungen Narbe bedeutend grösser ist, wie die absolute Zahl der Kerne in derselben Narbe einige Zeit spater.

Die Minderzahl könnte nun entweder dadurch zu Stande kommen, dass Zellen aus der Narbe fortwandern oder aber dadurch, dass sie an Ort and Stelle sich derart verändern, dass thr Zellcharakter nicht mehr zu erkennen ist.

Die Veränderungen, die wir die Kerne erleiden sehen, die Abnahme der Grösse und Tinctionsfähigkeit, ferner der Zusammonhang aller dieser Bildungszellen mit den neugebildeten Fasern bewegen uns zu der letzteren Annahme, und machen eine Wanderung im höchsten Grade unwahrscheinlich.

Es geht also danach der grösste Theil der Zellen des Graunlationsgewebes in den Faserzustand über, während ein anderer bedeutend kleinerer Theil anf einer gewissen Cebergangsstufe zur Easerbildung stehen und somit als Bindegewebskörperchen athalten bleibt. 
Fassen wir nun noch einmal die Resultate unserer Untersuchungen über die Heilungsvorgänge bei aseptischen Schnittwunden menschlicher Haut zusammen:

I. Die Verklebung der Wundränder findet nicht durch ein Exsudat, sondern direct durch eine Quellung der Wundränder statt. Das fibrinähnliche Maschenwerk, welches die Verklebung bewirkt, entsteht aus Umwandlung und Aufquellung des Cutisgewebes.

II. An der Heilung betheiligen sich durch indirecte Kerntheilung die Gefässendothelien und die Bindegewebskörperchen, mögen diese nun wirkliche Zellen oder protoplasmalose in der Grundsubstanz liegende Kerne sein. Zu einer irgendwie erheblichen Mitosenbildung in der Cutis kommt es vor Ende des zweiten Tages nicht.

III. Die sogenannte kleinzellige Infiltration kommt durch Vergrösserung der präexistirenden Bindegewebszellen, ferner durch eine Rückbildung der Grundsubstanz zu Kernen und Zellen, nicht durch eine Emigration von Leukocyten aus dem Blute zu Stande. An der Umbildung zu Zellen betheiligen sich sowohl die elastischen Fasern, als auch die leimgebenden Faserbündel.

IV. Ein Theil der den Leukocyten ähnlichen Elemente entsteht aus Kernen, in denen die Chromatinsubstanz in dieser eigenartigen Weise angeordnet ist. Alle diese Elemente sind einer weiteren Entwickelung făhig, und können an der schliesslichen Narbenbildung theilnehmen.

V. Die Bildung der Grundsubstanz in der Narbe wird nicht durch eine Ausscheidung eines todten Materials von seiten der Zellen, sondern durch directe Umwandlung der Zellen in Faserbündel bewirkt.

\section{Erklärung der Abbildungen.}

Tafel VIII.

Fig. 1. Präparat aus der Umgebung einer $3 \frac{3}{4}$ Stunden alten Wunde. Man sieht in den dunklen elastischen Fasern und Bändern kleinste deutlich roth gefärbte Chromatinkörnchen. Auch in der leimgebenden, gelb gefärbten Substanz sind kleinste Kernanfänge zum Theil wie bei $\mathrm{N}$ in leukocytenähnlicher Anordnung. Oelimmersion. 
Fig. 2. Hus der Umgebung einer 24 Stunder alten Wunde. Neben eiram durch Alkoholschrumpfung erweiterten. Spalt liegen in der Grundsubstanz ebenso wie auch weiter inmitten der derbea Bindegawebsbündel Kerne mit zerklumpter Ohromatinsubstanz vom Iypus der Leukoeytenkerne. Die Gruadsubstanz ist besonders noben dem Spalte sebon etwas erweiebt und gekörnt. Die Formen sind schon grösser als wie in Fig. 1.

Tig. 3. Aus der näberen Umgebung einer 38 Stunden alten Wunde. Neben einem durch die Härtung verbreiterten Spalt liegen in der schon aufgequollenen, etwas belles sich farbenden Grundsubstanz Ohromatinklümpchen zum Theil zu Leakocytentypus zusammengelagert. und $\mathrm{E}$ sind kleinste Ternanfänge vom Typus der Endothelkerne. T bezeichnet leukocyteähnliche Kerne, die sohon einen Hof orweichter Grundsubstanz um sich haben. Bei $\mathrm{F}$ siebt man die Grenzen der Bindegewebsbindel fibrinoid aufgequollen und roth geârbt. Die rotben, ein Netzwerk bildenden Fäserehen zeigen ubberall Kernanschwellungen, umschiessen aber moist ruhende, gelb gefärbte Grundsubstanz, el bezeichnet elastische Fasern mit Kernanfängen.

Wig. 4. Aus der Umgebung einer Wunde vow ersten Tag. In einen Geäst verschieden breiter, elastischer Fasern sieht man kleinste Chromatinkörnchen und Nucleoli, die zum Theil lealocytenähnloh angeordnet sind und zu theilweiser Erweichung der elastischen Fasera gefübrt haben. Man achte auch axf die fadenformig angeordnote Chromatinsubstanz bei $\mathrm{F}$. In dey rechten Halfte der Abbildung sind die Ausläufer der sogenamiten "Fibrinfäden" abgebildet. Die derben, im Ganzen noch ruhenden Bindegewebstọndel sind durch rothe Grenzlinien von einander getrennt. Diese tragen überall KernanschwelJungen, die theilweise nach Art der Nucleol (N) dunkelroth gefärbt sind oder wie bei $\mathrm{E}$ den Typus der Endothelkerne darbieten. Dort, wo wirkliche Spalten im Gewebe-sichtbar sind, wie bei S, zeigt sich deutlich, dass die Kernehen in der Grundsubstanz der einen oder anderen Seite liegen. Die Kerne sind neben den Spaiten zu Längsreiben geordnet und imponiren deshalb dort, wo sie schon etwas grösser geworden sind und einen Rof erweichter Grundsubstanz um sich haben, ganz als "wandernde Leukocyten" (W).

Fig. 5. Aus der Reactionszone einer 24 Stunden alten Wunde. An der Grenze drejer derber Bindegewebsbindel liegt ein grosser endothelialer Kern von körnigem Protoplasma umgeben, das mit seinen verschiedenen Ausläufern in verschiedene Bindegewebsbündel übergeht. Links in derben Bündel und recbts oben mehrere zerklumpte Kerne in Förnigem Protoplasma.

Wig. 3. Aus der Ungebung einer Wunde rom 10. Tage. In einem System elastiscber Fasern liegen schlanke Endothelkerne mit gefürbter Kernmembran und Cbromatingerüst. Bei $\mathrm{M}$ enthält eine derartige, in die Faser auslaufende Zelle eine Mitose. 
Fig. 7. Aus der Reactionszone einer 24 Stunden alten Wunde. Das Gewebe ist ausserordentlich reich an elastischen Fasern, in und an denen z. B. bei $\mathrm{N}$ zahlreiche Kernanfänge sind. Bei $z$ besteht eine vorgeschrittene zellige Umwandlung des Gewebes, einzelne Zellen sind aus dem Schnitte ausgefallen und haben ihre Abdrücke im Gewebe hinterlassen. Um die Kerne ist die Grundsubstanz schon vielfach körnig erweicht.

Fig, 8. Uebergangszone der fibrinoiden Verklebungsmasse zu ruhender Grundsubstanz aus einer 2 Tage alten Wunde. Man sieht von links oben nach rechts unten verlaufende dickere Fibrinbalken, die durch kleinere $Z$ weige sich verästeln. In den so entstehenden Feldern liegen bier 48 Stunden nach der Verletzung schon ausgebildete Kerne, meistens endothelialer Art, mit bellem blassem Zellleib. In den dickeren rothen Balken liegen kleine Kernchen (E). Nach rechts bin laufen die Aeste dieser rothen Fasern in das mehr ruhende Gewebe und gehen allmählich in die ungefärbten Grenzen der Bindegewebsbündel über. Die grossen, normaler Weise vorhandenen Bündel werden durch eine Reibe von Nebenästchen in kleinere Felder zertheilt. Dort, wo mehrere Bündel zusammenstossen, liegen grosse Endothelkerne, um die herum die Grundsubstanz in ein Gewirr feinster, roth gefärbter Fäsorchen aufgequollen ist $(G)$.

Fig. 9. Aus den tieferen Theilen der Cutis in der Nähe einer 24 Stunden alten Wunde. Zellige Schmelzung des Gewebes ganz entsprechend der eitrigen Schmelzung. In der mehr rubenden Substanz rechts sehen wir äberall kleine Kernanfänge (a und e), die, je weiter man nach links geht, an Grösse zunehmen und dem entsprechend einen Theil des Gewebes einschmelzen. Ganz links sind aus den Kernen nun schon wirkliche Zellen geworden, die aber noch an einer Seite mit der Grundsubstanz in Verbindung stehen. Von der Grundsubstanz bleibt schliesslich nur noch ein Rest von Balkenwerk über, das die einzelnen Zellen umschliesst und gleichsam jeder ein eigenes Fach anweist. K sind Kernanfänge in elastischen Fasern. 